\title{
The Principles of Causal Conspiracy
}

\author{
Michael M. Anthony ${ }^{1,2}$ \\ ${ }^{1}$ Department of Engineering, Fourah Bay College, Freetown, Sierra Leone \\ ${ }^{2}$ Enertron Inc., Hohenwald, Tennessee, USA \\ Email: uinvent@aol.com
}

Received 25 June 2014; revised 3 August 2014; accepted 1 September 2014

Copyright (C) 2014 by author and Scientific Research Publishing Inc.

This work is licensed under the Creative Commons Attribution International License (CC BY). http://creativecommons.org/licenses/by/4.0/

(c) (i) Open Access

\begin{abstract}
The human mind understands logical processes and causality and formulates theories based on logical descriptions of empirical evidence. The Principles of Causal Conspiracy is based on defining information as logical charges similar to electric charges. Such information charges can be modeled in the vacuum of a quantum probability firmament as symmetry of quantum charges with a zero net charge. Observation of a state lifts one of these charges in a Möbius transformation from a multipolar field of possibilities that maximizes a local monopole field that is observable. In the first of several papers, I introduce new and profound principles, the Principles of Causal Conspiracy, to provide a consistent epistemology for quantum theory, relativity theory and all the known sciences.
\end{abstract}

\section{Keywords}

Special Relativity Theory, Dipole, Monopoles, Quantum Theory, Quarks, Space, Time, Moments, Big Bang, MOND, Annihilators, Creators, Fields, Charges, Logic, Möbius, Lagrangian, Blackholes, Riemann Sphere, Causality, Uncertainty, Blackbody Radiation, 8-Fold Way

\section{Introduction}

The Principles of Causal Conspiracy are based on the observation that everything in nature is subject to a description based on the information obtained in a classical logical continuum from unobservable non-classical states. Quantum information and quantum states are known to come in conjugate states and sometimes in multipolar states. These generally represent the possible information states a quantum measurement can take. In a rudimentary sense, they are multipolar information states in the vacuum tied together in symmetry by fields that make their quantum information null or zero. Classical states generally have unipolar configurations that can only be in one state or another. Disturbing the symmetry of the vacuum by measurement results in a violation of a profound principle of nature is revealed. Such quantum information (i.e. illogical, non-classical information), 
can also be modeled by the same causal laws that govern classical logical information states. The two types of information (classical and quantum information) stand side by side as Möbius transformations [1] of one another. The Möbius transformation of the variable $z$ is given by a mathematical expression of rational functions:

$$
f(z)=\frac{a z+b}{c z+d}
$$

Definition 1: An observer is an instrument (or mind) that interacts with a symmetric quantum field to lift privileged logical information from multipolar illogical information in the quantum probability firmament.

Definition 2: Quantum information is non-privileged information tied in symmetry; classical information is privileged information obtained from a broken symmetry.

Definition 3: Privileged information is known information accorded a definite state; non-privileged information is tied in quantum symmetry.

The interaction between mind and matter involves the transfer of information from matter to mind to obtain structured information that is logically consistent (see Figure 1). If the information is illogical, the mind does not quite understand it.

- An observer does not need to be a human. Any physical interaction can disturb a vacuum symmetry to obtain privileged information. When information is created a privileged state is created. Thus all information that is classical is privileged since it must come in one specified state or another and specifying a state that had no particular privilege gives it a privilege.

- A privileged state in a space of $d$-dimensions can be symmetric in $(d+1)$ dimensions. There are many types of privileged information states, and these can all be summarized as bounded states in some higher dimensional configuration.

- The existence of a privilege can be modeled as the existence of truth charges. Suppose a system of dipolar truths exists in a small region of a logical space field. Such a system has zero truth charge with no privileges. If one should disturb this system by a measurement, one creates privileged information that did not exist before.

- The inconsistencies of the non-privileged truths in the system cannot be permitted by a formal logical continuum. The existence of classical and causal realism demands the existence of a field that either reduces and annihilates the truth charges as unobservable states in a dipole field or reduces the dipole field to a monopole truth field within the classical causal continuum.

- Logical information is privileged information that does not survive the existence of a conjugate statement. Thus an electric charge will not survive with its opposite charge. All dipolar charges can be modeled as logically consistent systems when they can be isolated as monopoles. A logical charge must have a conjugate.

Definition 4: Privileged information in a logical continuum is unbounded observable information.

By unbounded information I mean information that is restricted to certain axioms, conditions, motions, values or modes. If information is obtained from the vacuum of possibilities, it is privileged. Thus non-classical quantum information has boundaries that can be the result of knowing something, axioms, rules, fields, physical barriers and other special information. Bounded information forms Casimir traps. We tend to think of Casimir traps (see Figure 2) as two bounded plates that hold and reflect integer multiples of wave nodes. One can extend this concept of Casimir traps to all types of privileged information states. When a privilege is created, it must exist as a truth in the entire logical continuum. Privileges are generally accorded to distinguish between things, thus a boundary of axioms results in a sort of Casimir state for the information that is symmetric.

If one set of privileges is given to a particular state, then another distinguishable state may deserve a different set of privileges. In general, any time one gives a privileged status to something, one has distinguished it from all other things, and one has unbounded it from some information quantum trap. Things can share privileges, but when they do they form a class under the privileges they share in common. One gives privileges to distinguish one set of things from another. In a causal continuum once a privilege is created it is open information that all 


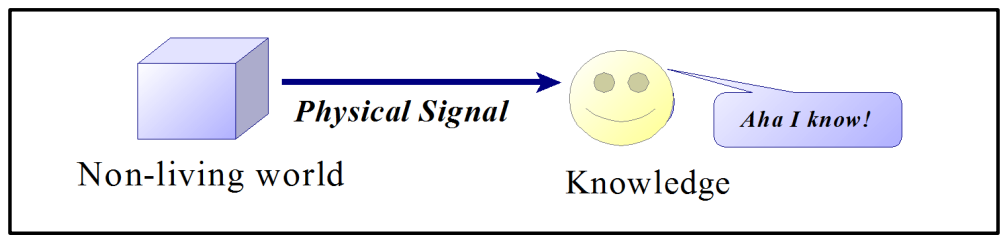

Figure 1. An information state is realized as a classical state by an observer. To do that the mind must somehow collapse the wave function and generate a monopole truth charge.
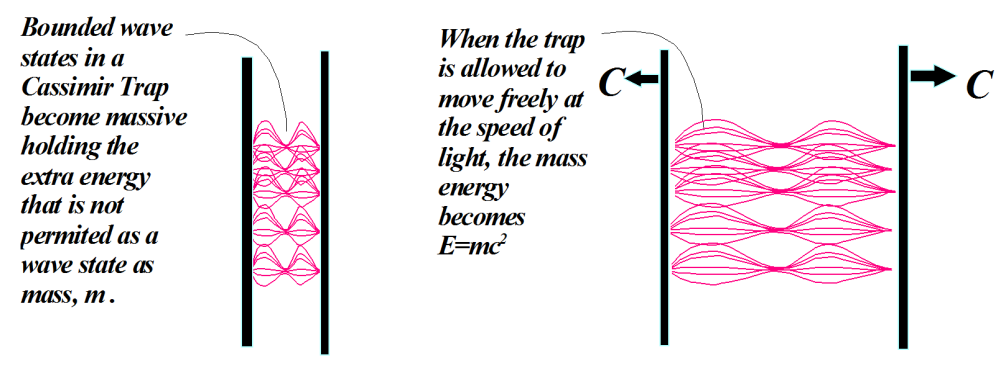

Figure 2. A typical Casimir trap with bounded information. The information waves outside the trap constitutes an annihilation field for the trap that causes the walls to attract. If there are no information waves outside the trap, it will expand and self-annihilate to fill up the entire logical space with the trapped information.

observers can interact with. If one has no information, then that information is non-privileged information. A secret for an observer is non-privileged since a secret is only known to a few privileged persons allowed within the axioms of the secret. Non-privileges have a boundary of some sort that reflects the axioms or wave functions of conjugate states and forms a closure around the information to make it unknown. So in a sense, every boundary binds symmetry of states of some sort. The fence around a garden binds a privileged place that excludes others from entering and interacting with the garden even though one could see through a fence to get some information one cannot interact with its contents. The canned tuna has no privileges with respect to its outer environment and cannot be tampered with until the container is opened for consumption. Privileges hold a special place in our world and provide us with a means of making certain things special for certain reasons.

Principle A of Causal Conspiracy: Nature abhors privileges.

Nature does not like to make the Earth the privileged center of the universe, and so Nature does not make anything or any place privileged. One can gain privileged information by disturbing symmetry. One can give the sides of a cube the non-privileged status of being symmetrical and equal, and by so doing information about the sides of the cube is trivial without measurement. Our minds seem to be able to assume privileges from symmetry alone and this can result in a falsity. One can gain privileged information from quantum theory by measurement to obtain a classical result. For example, by using symmetry one can infer that if a particle and antiparticle are created, they are perfectly symmetrical. If one knows something about one particle using symmetry, one assumes that one can gain information about the same state for the antiparticle without the need for measurement!

Broken symmetries carry a special privilege that seems to be allowed by our ability to logically conclude that the world has opposites that are equal and that gaining information about one state in symmetry can completely tell us what we need to know about the opposite state without taking the effort to measure. If you give a cube to a child who knows nothing about geometry and ask them to use a rule and measure its sides, they will innocently measure all sides. Each side of the cube has a length that is a mystery that has to be uncovered by the child (see Figure 3). However, if you teach the child about symmetry of the cube, it becomes complacent and just measures one length to determine all the dimensions of the cube. This lost innocence is due to the fact that our minds understand what symmetry is and one can make conclusions from symmetry alone about things one never 


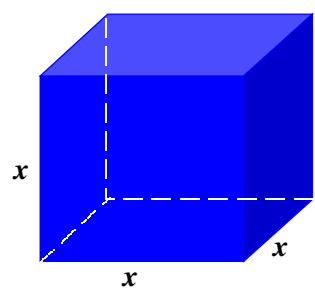

With a cube, we only need to know one measurement of a side and then we automatically know the others by symmetry. Given a side, $x$, the volume $v=x^{3}$ can be satisfied by one real value or two complex conjugate values. We leam something about the cube without measuring. This is the same as a spooky effect in quantum theory, where we know something about a distant symmetric object without a physical signal by measuring some property of its symmetric partner.

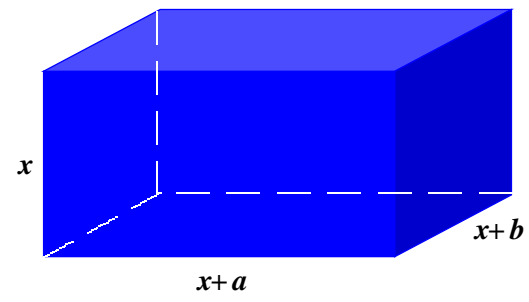

With a rectanguloid body, we need to know every side and to do this, we need real measurements. We cannot learn something about the rectanguloid without measuring. Given one side, $x$, the other sides can only be deduced as either greater or less than the real number $\mathrm{x}$, and no complex fields appear.

Figure 3. A simple cube can be specified using information about only one side and symmetry. However, there are always complex valued solutions to a cubic.

measures. One finds that there are also complex-valued conjugate solutions to at least two sides of any cubic-relation of symmetry. For example, consider the relations that satisfy:

$$
y=x^{3}
$$

There is a real-valued solution and two complex-valued solutions that are conjugate fields:

$$
x=\sqrt[3]{y}, \quad x=-\frac{y^{\frac{1}{3}}}{2}(1 \pm \mathrm{i} \sqrt{3})
$$

These complex-conjugate valued solutions show that the symmetric information gained by what one thought could be a single real measurement is not so true. Thus a cube-symmetry like any other physical symmetry has a quantum mystery associated with it! Anytime one makes a measurement or an observation of some state, a privilege is created that makes one know something about the world. Thus, every time we measure or observe, we are generating some class of privileges in nature that were not there before.

In general, privileges keep information as unbounded wave states outside a sort of Casimir trap. However, as I have said, nature abhors privileges, so such privileges as accorded by an observable world demand the existence of conjugate worlds of symmetry binding the information in a reflective trap that does not allow the waves to pass to the outside or new waves to enter the privileged area. This means that such privileged information can generate attractive or repulsive fields of some sort like a Casimir trap, just as charges attract and repel each other. The observable world must be dual in nature. A privilege exposed to the universe of observers in a logical continuum generates a causal structure for all the space it is exposed to. Thus every observer will see the same privileged result given enough time. If the cat is dead for one observer, it is dead for all observers. This violates the Principle A of causal conspiracy. Nature abhors privileges. How does nature eliminate a privileged information state that has been created by measurement? Here are examples of privileges that have been described before by various important scientists and mathematicians.

\section{Privileged and Unprivileged Information States}

\subsection{Einstein Privileges}

What Einstein discovered as the Principle of Special Relativity [2] is that the information one gains by measurement using a physical signal cannot give any observer privileges over other observers. Any privileged information gained cannot be deemed absolute in nature! This is the core difference between quantum theory and relativity theory. Nature abhors privileges and all observers must obey this law of invariance. The relationship between quantum theory and special relativity theory can be summarized as follows:

1) Relativity theory deals with privileged information obtained from the vacuum, i.e. measurements that we have made from prior unknowns with privilege; and 
2) Quantum theory deals with non-privileged information, i.e. information that still lies in a symmetric state in the vacuum without privilege.

When Einstein said there are no privileged observers in nature, what he said could be extended to a far deeper result than just a notion of invariance. This new notion is not just about light and observers; it is about knowing something, and that includes anything!

\subsection{Symmetry Privileges}

Experiments in quantum theory that can give privileged information as "spooky action-at-a-distance" that relies on some symmetry condition that is treated as absolute. Such privileged information is not allowed by special relativity theory. A symmetric class of particles forms a non-privileged class that excludes outside intrusion into its states. Imagine the symmetric system to be enclosed in a small region of some logical space continuum. One can imagine a sphere that can have one of two color states for simplicity. Before observation, the observer does not have privileged information about the color of the sphere. The color matrix is tied in a dipole field in a quantum region that is allowed to be illogically both colors. This region is an illogical region in a logical continuum. The symmetry of matter and antimatter in quantum experiments starts from such bounded dipole states that form Casimir traps. Such symmetric systems are illogical and non-causal structure in a logical spacetime environment. Their behavior will confound logic. Expanding this region to larger areas simply dilutes the symmetry further and imposes mutual classical exclusion of the colors. The observers can now observe a separation of distinct color states in different regions provided they are far enough apart not to interfere with the laws of causality. One cannot see both colors at the same time! Any measurement of such a system demands that the symmetry be imposed as acausal.

When one claims that one can know something using symmetry, one must be cautious about conventional notions of symmetry. One must reject such a statement and believe that even information concerning absolute symmetry cannot be accorded a privileged place in nature. In fact, the entire theory of special relativity could be recast in the light of the new and simple Principle A of Causal Conspiracy. Mirror symmetry has flaws in a causal spacetime structure (see Figure 4).

In the real world, our minds can infer "truths" from logically consistent deductive reasoning. However, deductive reasoning relies on causality and causality in turn relies on ordered information sets. Symmetry in the real world is not what we think it is. Symmetry does not give us a right to say we know things without measurements. If one knows one thing about a state in symmetry one does not have the right to say one knows other things about it unless one can deduce these facts using axioms other than symmetry. One cannot say that because of symmetry between parallel lines one knows that they never meet. There are no axioms within 3-D Euclidean geometry that one could use to prove this. One needs a higher dimensional geometry to discover the falsity of

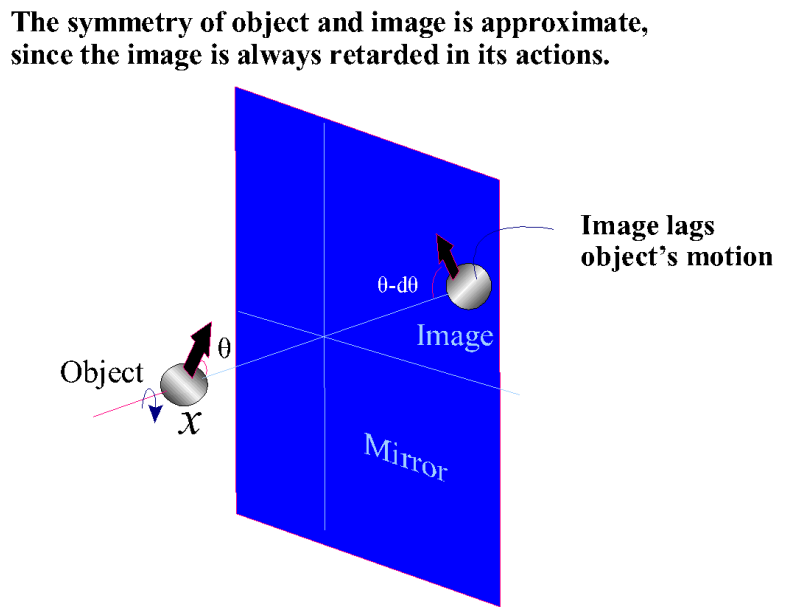

Figure 4. In a conventional mirror, symmetry is broken by causality. The ordering of information between the image and the object implies causal order and the image motions are retarded with respect to the actual motions of the object. Causality is a result of broken symmetry. 
such an assumption. Every time one encounters symmetry, one seems to think that one could infer results from it without actual proof. It is important to note that any disturbed symmetry will propagate a result to infinity to fill the space continuum with a logical outcome. In later papers, we will explore the reason why straight lines in 3-D space meet at infinity.

When one knows the state of a particle's spin, we must not conclude that the antiparticle has an opposite spin without actual measurements. Actual measurement implies a time ordered causality since such symmetric systems represent conjugate systems that are incompatible without time ordering. If one does experiments to prove this one ends up with spooky results that baffle our logical minds. Nature does not allow us to simultaneously specify multiple measurements without some repercussions. There is always some annihilating information field that will disturb such simultaneous measurement in nature. Symmetry alone cannot give privileged information that one does not gain by means of an actual measurement using a physical signal [3].

\subsection{Kurt Friedrich Gödel Privileges}

Kurt Friedrich Gödel [4] was a famous logician and mathematician who influenced many areas of modern mathematics and modern physics. In a famous theorem called the theorem of incompleteness, he stated that:

"No consistent system of axioms whose collective theorems can be listed by an effective and consistent procedure that can prove all truths about the relations of its elements, and such an axiomatic system cannot prove its own truth, and it is incomplete."

I will state this in another way:

"No logical continuum can hold all truths about the relations of its quantum symmetries and such a logical system is incomplete."

Gödel's theorem is an example where one finds that whenever truth or knowledge is confined to a finite set of privileged axioms, there is missing information that cannot be inferred by such privileges. Again mathematics abhors absolute truths. Whenever an axiom is chosen, a privileged class of information is created. If the information were not privileged, it would not be classified as different from any other information we can gain. By making a statement that isolates or collapses a set of truths or axioms, one is lifting such statements from a confined non-privileged status and there are always conjugate truths that are missing. The same thing happens in mathematics as in nature.

\subsection{Casimir Privileges}

Hendrick B. G. Casimir, a physicist, [4]-[6] discovered that when two parallel plates are really close to one another, the plates attract one another generate a force field. The plates eventually meet, and the gap reduces to zero. The force created is called a Casimir-force (see Figure 5). Extend this notion to any contained set of in-

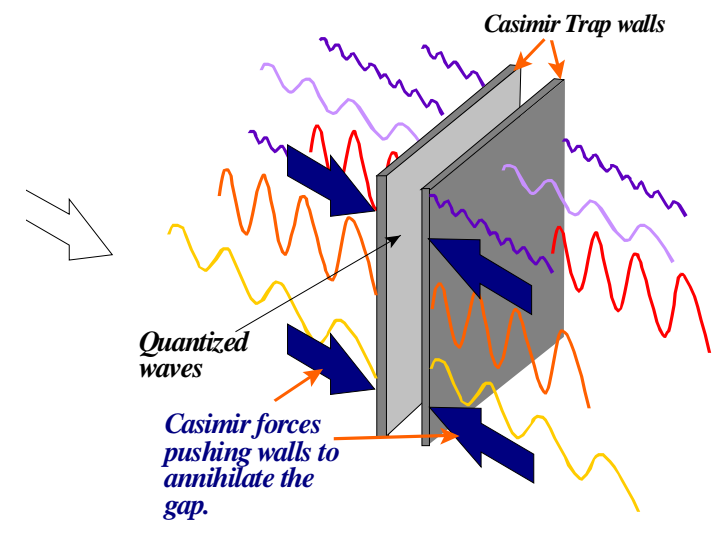

Preferred information is created when a trap included only certain prefered frequencies. It is like tuning a radio to a certain set of frequencies. The rest of the frequencies revolt and try to annihilate the preference as a symmetry break!

Figure 5. Privileged information can be annihilated by nature to an unprivileged class. The Casimir trap confines privileged information and nature generated fields that want to annihilate the privilege. 
formation fields. When information waves are confined inside an information trap with perfectly reflective walls to the information waves, force fields are created between the walls that want to annihilate the trap. The trap contains a privileged class of information waves. In this case, one finds that the symmetry of the system outside the trap has been violated! Instead of having waves of all wavelengths, such a trap will only have waves with frequencies that are integer multiples of some fundamental frequency. Nature abhors such privileged information classes and so forces are generated to annihilate the privileged trap. Note that the walls attract and this is what dipole charges will do to annihilate their quantum charges.

\subsection{Quantum Privileges}

Quantum measurements are plagued with uncertainty relations. Just when one thinks one can measure the velocity (time related) and position (space related) of a particle, one finds that the accuracy of the measurements is inherently compromised by quantum uncertainty. One cannot have the privilege of measuring both quantities at the same moment.

\subsection{All Privileges}

Information that is created always has annihilated conjugate information states. For example, whenever one creates information that tells one that the answer is YES, there is a NO answer that is annihilated [7]. One cannot know both answers at the same time. One does not have the privilege of being omniscient. If an observer gains information about a physical state or a mathematical object, there always exists information that is annihilated by the same experiment. To justify absolute information, one must generate inconsistencies (Gödel) in the information field. If one says, "I know from symmetry that such and such must be true," one must be cautious and suspicious of such privileged information being deemed factual without making a real measurement. I want to elevate this principle of privileged information to a higher set of principles of nature I refer to as Principle A of Causal Conspiracy.

\section{Information as Observable Classical Monopoles and Unobservable Quantum Dipoles}

I state the following principle:

Principle B of Causal Conspiracy: Knowledge gained is a Möbius transformation of the Quantum Probability firmament from a multipolar illogical state to a monopolar logical state and it obeys Lorentz gauge invariance.

From this principle one can deduce that every unobservable dipole field is associated with an observable monopole field.

- Every observable monopole electric charge is associated with an unobservable pair of dipolar magnetic monopole charges.

- Every observable monopole space field is associated with a dipole moment time field with past and future monopole charges.

- Every observable monopole truth charge is associated with an unobservable dipolar quantum information monopole charges.

- Every observable monopole, (unidirectional), expanding field such as the expanding space field of the universe is associated with unobservable dipolar gravitational monopole charges.

- Every observable monopole spin charge is associated with an unobservable dipolar quantum spin-up spin-down charge.

- Every weak charge (decomposition of fractional quark charges into constituent states) is associated with unobservable strong attractive quark gluons.

One can imagine that each dipole pair of charges of a dipole field can be Möbius-transformed from a neutral quantum-paired state of zero-information-charge to a classically observable charged monopole field state, i.e. the collapse of the wave function (see Figure 6). One can chop a dipole magnetic field into smaller and smaller bits and still get dipoles and never get a monopole field. Similarly one can chop moments in time into smaller 
and smaller bits and still get moments with future and past charges. One can chop a gravitating body into smaller and smaller parts and still get a gravitating body. But the electric field, the space field, and the expansive fields are all monopole fields that extend to infinity. The process of logical information creation involves a competition of monopole states in an illogical dance and with the winners expressing their charges as observable states (see Figure 7).

\section{The Concept of Maximization Fields and Minimization Fields}

Unobservable quantum dipolar or multipolar information fields are minimized states. Left alone, they are unobservable and have a zero or null information charge. One can look at these states as minimized stated that can annihilate each other. When maximized, they can form privileged observable monopole states with charges. These monopoles charges are collapsed states that can generate a sort of Casimir field. Möbius transformations of minimized fields of a dipole state of null quantum information result in classical observable charged monopole states. These charged monopole fields want to annihilate privileged information states. The annihilation forces maximize the states into infinite or expanding monopole fields that are observable as classical charged states in a logical-classical continuum. When a measurement disturbs symmetry of information in the vacuum, it creates a situation that allows one to probe the illogical realm of conjugate states coexisting at the same place at the same moment. Such a situation must satisfy two conditions in a logical-classical spacetime continuum:

1) The conjugate states annihilate back into the vacuum as dipoles without a result that is illogical.

2) The conjugate states separate into distinct logical continuums that cannot be observed or measured simultaneously to generate illogical information.

3) Process 1) is a minimization of the states, and process 2) is a maximization of the states.

- The lifting of information from the vacuum, otherwise known as the collapse of the wave function in quantum theory, results in maximization of a privileged state by a Möbius transformation of an illogical dipole field into a logical monopole information field with a truth-charge. The unobservable dipole fields are illogical and mutually exclusive conjugates. In order for the human mind to gain information and understand it, it

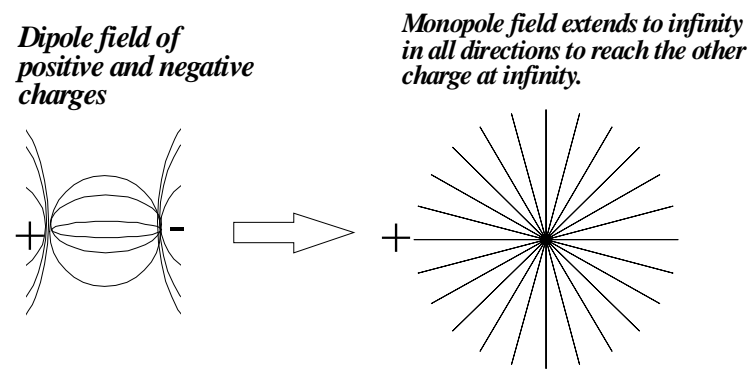

Figure 6. Shows how a dipolar field can be changed to a monopole field by observation and measurement. The dipole field is unobservable at the quantum level, but it is made classical when the monopoles are separated to observable states.

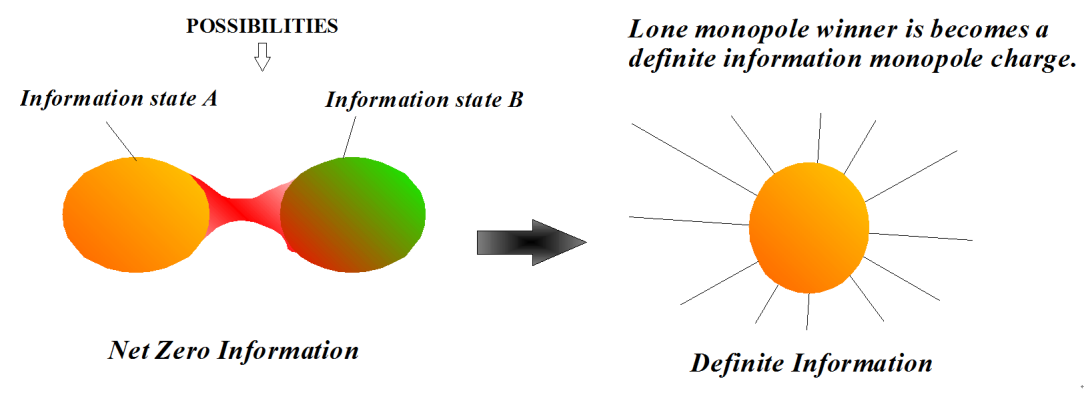

Figure 7. Shows how the human mind somehow interacts with nature to maximize dipoles as monopole fields with definite charges. Could electrons and positrons and other quantum charges be some type of specific information charges that were maximized from the vacuum? If so what mind or observation state made that happen? 
must lift a monopole field of truth from the vacuum of multipolar possible truths and maximize a privileged monopole field as an observable truth-charge that fills the logical continuum of the spacetime field of observation. When one wants to know if the answer is YES or NO , one collapses a result by Möbius transform ation of the unobserved information paired YES-NO charges by taking one charge to spatial infinity and leaving the other local to get a monopole information field such as either a YES or a NO.

- The expansion of the Universe is Maximization Annihilation of all information created in the Big Bang. One can imagine the Big Bang as a collapse of monopole matter states from dipolar matter-antimatter quantum paired states. The universe expands and wants to clean itself into an empty symmetry of lone monopole charges. If one looks at the symmetry it is a dual space field of matter and antimatter sitting side by side in a higher dimensions and separated by a moment in time.

- This means that the Big Bang is a set of monopole matter states maximized from dipole symmetry of matterantimatter! It is no different from a YES-NO collapse!

- The process of maximization annihilation results in lone monopoles that becomes isolated from each other by infinite spaces. In other words, the universe wants to select every possible result by maximization. It wants to express in a time ordered manner, monopole space fields as truths all by themselves with conjugate states at infinity. Each truth must occupy an infinity monopole classical space without a conjugate.

- One can imagine the pair of information states sitting in a higher dimensional moment time dipole field and then transformed into an observable privileged monopole field by the mind or observer. Our minds are modeled to understand logic. This model is a result of the property of the spacetime field as an ordering field.

- Both maximization and minimization symmetries are represented by two parallel space membranes sitting side by side and separated by a moment in time. In this way a notion of a moment in time is replaced with the notion of minimized dipoles. One state of the dipole is in the past and the other state is in the future. This configuration allows the quantum state of the minimized symmetry to be lifted again when another observation is made at another moment.

- The gravitational field is a minimization field that annihilates information states back into the vacuum by eventually forming black holes. Mathematically, one can say that a blackhole is the limit of how many logical charges can be expressed in a given space field. Blackholes are like Casimir traps that annihilate information back to the vacuum [7].

- Similarly, the electric field is a maximization monopole information fields that rejects duality of like information charges while the magnetic field is a minimization dipole field that wants to crush a north magnetic pole into a south magnetic pole to annihilate the field back into the vacuum.

- To restore symmetry and abide by Principle A, matter and antimatter in this scenario will be separated into two conjugate maximization mirror image spaces by spatial infinity at each minimized moment in time. A moment would vary from point to point between such a mirror spaces depending on the matter-antimatter content (the extent of symmetry break).

Definition 5: Infinity is where all that is KNOWN IS NOT and where all that is NOT KNOWN IS.

\section{The Concept of Bosons and Invariance}

Invariance can be understood if one applies Principle A of Causal Conspiracy to bosons and logical realism.

Statement: Invariance is the way Nature has found to make the answer to all questions the same way for all observers [8].

$$
\phi_{\mu}(x) \rightarrow \mathrm{e}^{i q . \alpha(x)} \phi_{\mu}(x)
$$

where, $q \cdot \alpha(x)=2 \pi n$.

According to Principle A, when privileged information is created in a space field from the vacuum, there must exist conjugate information that has been removed or annihilated from the observation space. In order to restore the symmetry of the original states, these conjugate states can be envisioned as sitting at infinity in higher di- 
mensional symmetry (spacetime) on a parallel conjugate unobserved space field. The whole process represents a restoration of invariance of the privileges to moment dipole ordering in time as paired conjugate states in a higher dimension. In 3-dimensions, a monopole state looks like an infinite field in the past space field with no conjugate. However in 4-dimensions one can imagine its conjugate sitting in a dipole symmetry that connects two infinite space-sheets sitting side by side separated by a moment in time.

When one creates a pair of photons by minimization of opposite matter-antimatter states, one finds that the photon information fields maximize into separate continuums. One cannot use logical realism to observe both states at the same time. If one does that, one gets spooky relations. The speed of information bosons for a given revealed state is governed by the boson that gives that information. Thus, we can have many types of bosons for many types of information states. The YES-NO answer has bosons that the mind can read somehow. Any knowing has bosons associated with it. The bosons of an information monopole are unique to the type of information. For example there are specific bosons for spacetime information, spin information and so on. Mutually exclusive information states are separated by maximization at boson exclusion velocities that cannot be surpassed by a logical observer in a logical continuum (Einstein's speed of light for example). One finds that the speed of a boson is the maximum speed an observer cannot reach in a logical continuum for the specific information the boson carries.

If an observer can surpass the speeds of maximization of a boson of a particular information type, then that observer will experience conjugate information states and illogic! As shown in Figure 8, an Ising model can be used to demonstrate how the dipolar alignment looks in higher dimensions where the dual symmetry can be seen.

\section{The Birth of Logic and Classical Realism}

Imagine that one can probe the quantum world with a truth field of some sort. This field tells us if there is an opposite truth charge somewhere close by. Since the classical observable world is logical in nature, any observable state selected from a set of conjugate possibilities becomes a monopole charge in nature. One can imagine that the information is tied in the vacuum in a zero net charged state as a dipole pair. When one state is lifted, a charge appears.

Statement: A quantum charge expressed in a local region is a measure of the probability charge, and it is maximum when no conjugate charges exist in the local region.

Nature would make sure that we do not find a logical contradiction in the space field and so the two charges will always want to neutralize each other as if they are attracted to one another. If our minds or instruments are built to only give logical answers, when one searches for an answer, only one answer can be found in the entire logical space field. The contradiction is lifted if one maximizes the opposite answer and sends it so far away and so fast that one cannot communicate with this opposite answer and get a contradiction of logical results. Since information created is a break in perfect symmetry, there exist annihilating fields that want to restore symmetry. It is important to know that whenever one finds that information has been created from the vacuum, there will be an annihilating field that wants to annihilate this information back into the vacuum as in (a), or that wants to create a logical continuum. Every knowable property of a system is information that must conform to some logical description that one can understand. Logical observable states (outcomes) are exactly similar to monopole charges such as electric charges that have fields associated with them. The connection between modular functions [9] and logical systems is very deep and a clear understanding of this relationship can be made using geometric concepts.

Statement: A quantum charge expressed in a local region is a measure of a logical charge, and it is maximum when no contradictions exist in the local region.

A binary logic system is a relationship between two possible states with values normalized to probabilities of 0 and 1 . In the theory of causal conspiracy these represent the maximum relational charges between two states 


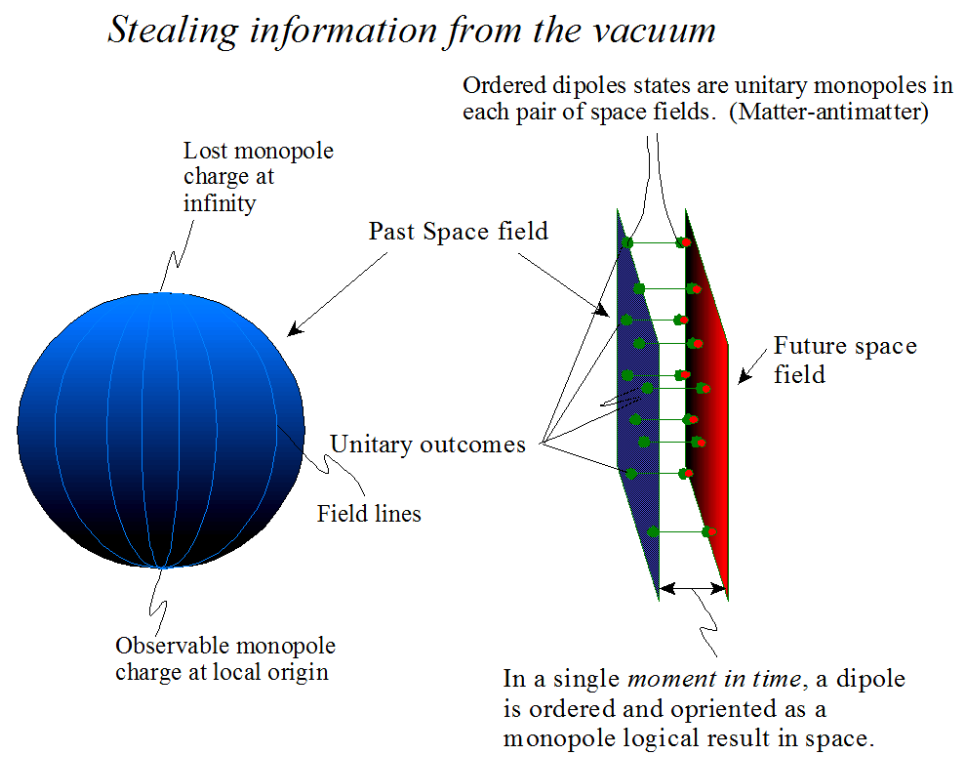

Figure 8. The universe can be viewed as a symmetric state with matter and antimatter states on separate logical space continuums. This is just like saying a YES answer is lifted from the vacuum symmetry of YES-NO and the NO answer is Möbius transformed to infinity on a mirror space a moment away. This can also be viewed as dipoles on the Riemann sphere.

of a binary logic system. Following Sikorski [10], for each pair of states in an extended 2-D space one can fill a lattice with binary state logic nets of Boolean algebra if

$$
\beta=\left(B, \wedge, \vee,{ }^{\perp} 0,1\right)
$$

here $B$ is a set of two truth elements designated as binary states of 0 and $1 \wedge$ (meet) and $\vee$ (joint) are binary operations on the set and, $\perp$, is a singularity operation on $B$. The singularity operation allows one to distinguish an element that acts on itself such that:

$$
a \vee a^{\perp}=1, \quad a \wedge a^{\perp}=0
$$

where all operations on $B$ satisfy the following identities for all $a, b, c$ in $B$

$$
\begin{array}{ll}
a \vee b=b \vee a & a \wedge b=b \wedge a \\
a \vee(b \vee c)=(a \vee b) \vee c & a \wedge(b \wedge c)=(a \wedge b) \vee c \\
a \vee(a \wedge c)=a & a \wedge(a \vee b)=a \\
a \wedge(b \vee c)=(a \wedge b) \vee(a \wedge c) & a \vee(b \wedge c)=(a \vee b) \wedge(a \vee c) \\
a \vee\left(b \wedge a^{\perp}\right)=a & a \wedge\left(b \vee b^{\perp}\right)=a
\end{array}
$$

The reader may want to consult the book The Structure and Interpretation of Quantum Mechanics by R.I.G. Hughes (Harvard, 1997). Two orthogonal states $A$ and $B$ can represent the possible combination as logic statements on a logic lattice generated with each lattice point carrying with it a set of relations that represents its contribution in a closed logical relation. This can be seen in the simple two-state diagram as shown in Figure 9. Interpret lattice diagrams in this theory as follows:

1) A vertex represents a logical monopole state;

2) A boson is represented as a line or edge connecting two vertices or states (or a loop connecting to the same vertex) and is an interaction between two states;

3) A field line is represented by an edge that connects a vertex state to infinity.

Thus a field line is a boson trajectory with a virtual boson that is infinitely extended. Orthogonal representations of symmetric states on the Riemann sphere represent binary logic states that divide the Riemann sphere into a pair of conjugate states with fields joining them. The binary logic states are either a pair of vertices con- 


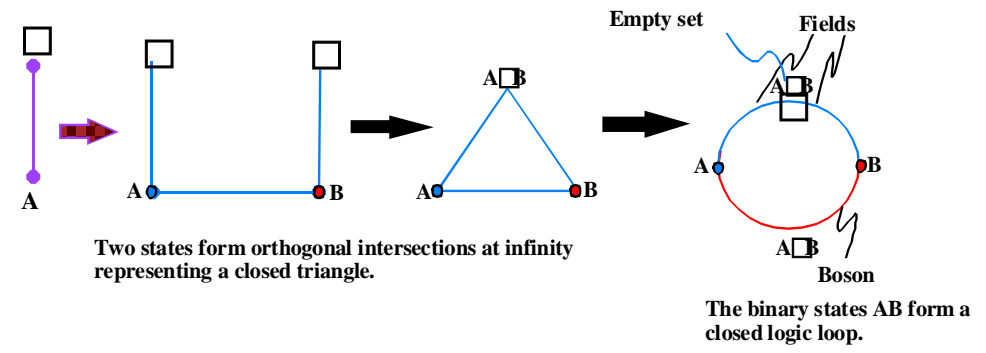

Figure 9. A logic diagram can be generated using the simple concepts of geometry. Field lines are infinite in extent. A two state logical system can be extended to a three-state relation at infinity to form closed geometric figures.

nected by a field or have bosonic interactions between them that nullify the charge that would otherwise be carried away by a unitary logic state. To fully understand how to determine the "net charge" of a relation one needs to learn how to make relational diagrams. The first rule is to create a fundamental graph relating the states showing relations mediated by bosons. For example for two quantum states $A$ and $B$ hare information by projecting bosons and fields. An observable state $A$ is associated with an unobservable state $B$ and the information field satisfies the two statements:

I know something about $B \uparrow$ (boson) and I do not know anything about $B \uparrow$ (field).

This is represented by a field-line from $A$ going to infinity.

In words one projects from $B$ the same set of statements:

I know something about $A \uparrow$ (conjugate boson) I do not know anything about B (conjugate field).

One can now regard any quantum charge as a measure of a logical charge in a quantum system. Thus, in any quantum system, information lifted as a monopole result will express its logical truth as a monopole charge of some sort.

In Figure 10, a dipole is shown with one monopole $A$, as a known state. The known state is a monopole charge that extends a field to the unknown state Bat infinity. This is represented as a field line going to spatial infinity as an infinite charge. $A$ is an observable monopole charge. In Figure 11, the interactions of $A$ and $B$ can be seen as fields extended to spatial infinity from $A$ and $B$, and can also be seen as a dipole field connecting the two states locally in a local region of finite volume. Thus a vertex state with an infinite edge connected to it must be interpreted as a logical monopole charge. This monopole charge cannot find a conjugate state to form a logical relation within a logical continuum $\Xi$, and so it keeps sending a connecting field until it reaches infinity where it connects with its conjugate charge.

Statement: Any observer query in a logical continuum of a quantum system results in a logical causal set of relations.

A virtual boson that cannot find a logical "solution" in a logical continuum $\Xi$ forms a charge field that extends to spatial infinity. If a boson finds a solution in $\Xi$ then the two states must be "oppositely charged states" with respect to their union since the field vectors of the bosonic statements must either emanate from $A$ and become a source that sinks at $B$ or emanate from $B$ and become a source that sinks at $A$. One associates the two states as charges that cancel with a net logical charge of zero. Thus one says that no knowledge has been gained. The reason charges exist is because our minds cannot accept two logical statements as consistently occupying the same region. If they exist they will appear as natural charges that want to cancel one another so that the charge inconsistencies are removed from the logical space field. In fact such a pair occupying a finite region will reduce each other's logical charge (electric-charge). The closer they come to one another the more intense the need to annihilate each other. On the other hand if the charges are like charges and they generate the same logical state at different spatial locations, they will repel each other so as not to occupy the same region. Eventually they both will occupy and infinite space without interfering with one another's logical charge. The objective principle is to eliminate contradiction of a logical space fields and a logical time ordering.

If each logical state has unit charge then one can build diagrams for null charges and singularly charged states provided one keeps in mind that a single state must carry a net integer charge that could be positive, zero or neg- 


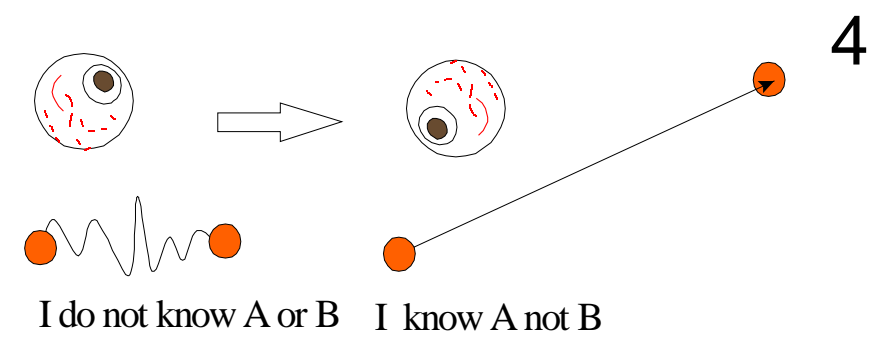

Figure 10. The human mind collapses the wave function of a state from a dipole pair of possibilities. This is like positioning an object at the focal point of a lens to send its image (conjugate) to infinity. Since all information has bosons associated with it, our minds act on these bosons just as our eyes act on light!

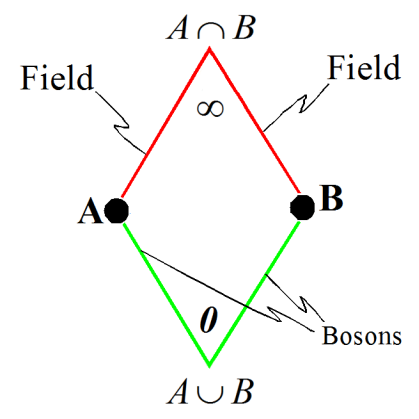

Figure 11. Shows a simple diagram showing how two states $A$ and $B$ are tied together by two bosons and two fields.

ative. In this representation one could partition and internalize the relationship between two or more states and form relationships between even closed systems. The same rules will apply for generic systems that form singular states as if the composite state is itself a single point in some abstract space of two or more dimensions. The relational lattice for the binary states is then the simple four-point lattice.

If a system were to form closed fields then one must make sure that all the states are represented by bosonic states that are orthogonal to the fields that meet at infinity. Expanding the logical relations to the two-dimensional Riemann sphere requires three orthogonal connections representing the three great circles of the sphere (see Figure 12). The intersecting points of these great circles result in states or vertices with a conjugate state sitting at infinity. There are six such vertices with each vertex having four edges connected to it. The number of edges connecting to a vertex is called the edge multiplicity $M_{E}$ of the vertex.

Any edge going to infinity will be regarded as a field from the state vertex it is attached to. The state at infinity is regarded as a full charge and so one can say that the field passes through infinity. One can then map these states on the Riemann sphere with infinity at the North Pole. One sees that there are six vertex states at the intersection of the three great orthogonal circles since they are either sources or sinks. Now as stated earlier a field is a line that goes to infinity. Thus there are six vertex states four field charges and eight bosons. These relations can be represented by the octahedron as six symmetry vertices. To see this clearly one makes what is called a Schlegel diagram of the octahedron whose dual is a cube as shown in Figure 13.

It is important to note that the $4 \times 4$ matrix below can summarize the full symmetry of Boolean logical operations of the two states $A$ and $B$ :

$$
\begin{array}{cccc}
0 & A & B & A \cup B \\
\bar{A} & \bar{B} & \bar{A} \cup \bar{B} & (\bar{A} \cup \bar{B}) \cap(A \cup B) \\
\bar{A} \cup B & A \cup \bar{B} & \bar{A} \cap B & A \cap \bar{B} \\
\bar{A} \cap \bar{B} & A \cap B & \infty & (\bar{A} \cup \bar{B}) \cap(A \cup \bar{B})
\end{array}
$$




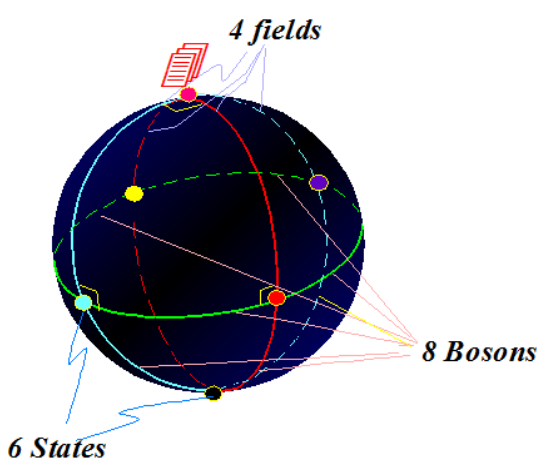

The orthogon al tesselations of th eRiemann sph ere lead to 6 states connected by 4 fields and 8 bosons

Figure 12. Shows the orthogonal primary states that can be formed on a Riemann sphere.

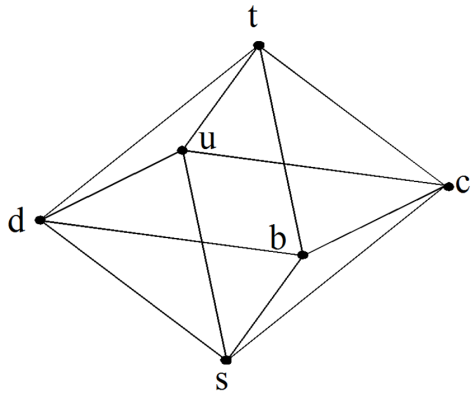

Figure 13. The fundamental logic diagram of two states tied together by two bosons and two fields.

This exhausts the possible relations between the two states $A$ and $B$. One then sees that these fill the Tesseract (4D hypercube) exactly. The Tesseract (4D hypercube) has 16 vertices 32 edges 24 faces and eight cubic cells. It is therefore clear that the 4D space has a special place in the algebra of logical states that cannot be expanded further. The binary logic continuum is complete in four-dimensions and is represented by a hypercube relation!

\section{Birth of Causality and the Space-Time Field}

When symmetry is disturbed, it either collapses back into the vacuum by minimization or it maximizes to generate monopole logical information in dual mirror spaces to restore the symmetry in a higher dimensional spacetime (of two sheets). The vacuum can be viewed as a mixture of dipoles in a net zero information state as shown schematically in Figure 14. The maximized representation in two sheets and the minimized representation as vacuum dipoles are two ways of representing the vacuum symmetry. When a logical ordering is made, information from the vacuum can be ordered by maximization to generate a pair of mirror image logical continuums that are mutually exclusive as shown in Figure 15.

Imagine that the vacuum is full of dipole pairs of states tied together with null-charge symmetry.

One can imagine these dipoles like Magnetic North Poles and Magnetic South Poles tied together in the vacuum in a random manner before observations are made. When the mind disturbs the symmetry, the dipoles become aligned and the entire vacuum aligns with one of these space sheets resulting as a past that is an observable result, while the unobserved states align on a future unobservable sheet. All of a sudden there is a condensation of dipoles that align on two sheets as shown in Figure 15. Maximization as I have said is the process of separating illogical states to form a logical observable privileged states in a logical continuum. Thus one can imagine that at each moment, one collapses an observable universe of monopoles while generating a future possibility by aligning the vacuum. This process gives us privileged information which according to Principle A, has conjugate states that must be annihilated in a four dimensional continuum. In three dimensions, infinity appears 


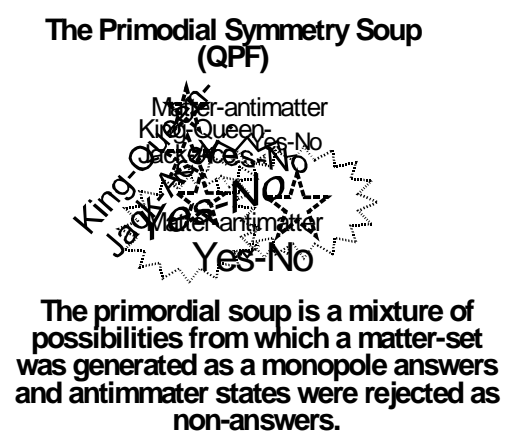

Figure 14. The primodial soup is a mixture of multipolar states that have no orientation or order. Disturbing this symmetry can cause the entire structure to collapse into ordered states similar to a Magnetic Ising model. One can think of the Big Bang as an ordering of monopoles across space and time two conjugate sheets.

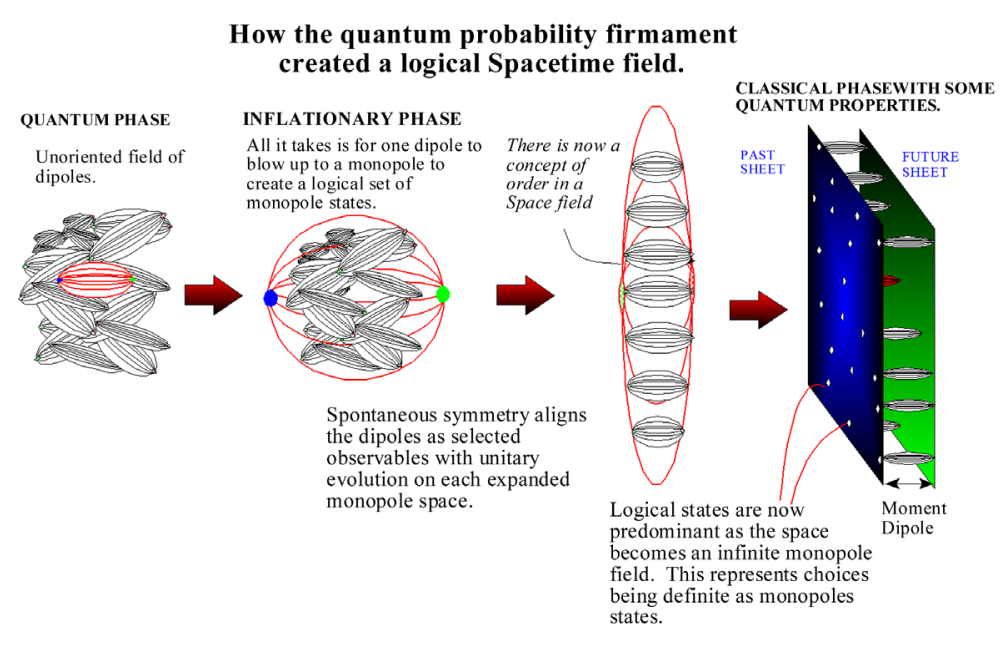

Figure 15. The Big Bang is an alignment of vacuum states into conjugate states that do not contradict logical realism. Each state generates a causally consistent and logical universe only one of which we experience at each moment in time.

to be where all the conjugates lie. At infinity one gets a symmetric mirror space populated by conjugate unobserved monopoles. Maximization sends conjugate unobservable states to infinity on a parallel sheet. Maximization occurs because the states in symmetry have no privilege and by disturbing the symmetry one has selected a privileged states over the mutually exclusive possible states. By locally disturbing the symmetry by measurement to generate an observable, the non-privileged state is annihilated and the states must be separated as independent logical outcomes in a logical classical space continuum. The unobserved state is taken to infinity where it cannot interact with the causal structure of a logical continuum of the observer and for it to restore symmetry; it exists on a parallel membrane.

Infinity can be a moment away (small radius of Riemann sphere) and yet so far away! So one can imagine that the universe has two parallel conjugate past and future spaces sitting side by side as parallel universes of conjugate information separated by a moment in time. One cannot see antimatter states because they are ordered a moment away at spatial infinity into the future of matter states. As long as conjugate states are separated by a causal continuum, privileged states can exist side by side in a dipole configuration in time. Infinity looks like the maximum distance away from us and it is just the Big Bang, where dipolar quantum states started as observables. As shown in Figure 16, the two mirror spaces form a conjugate set with space ordered and time ordered causally consistent results. Each moment is then represented as an instantaneous space of ordered states we call a universe. 


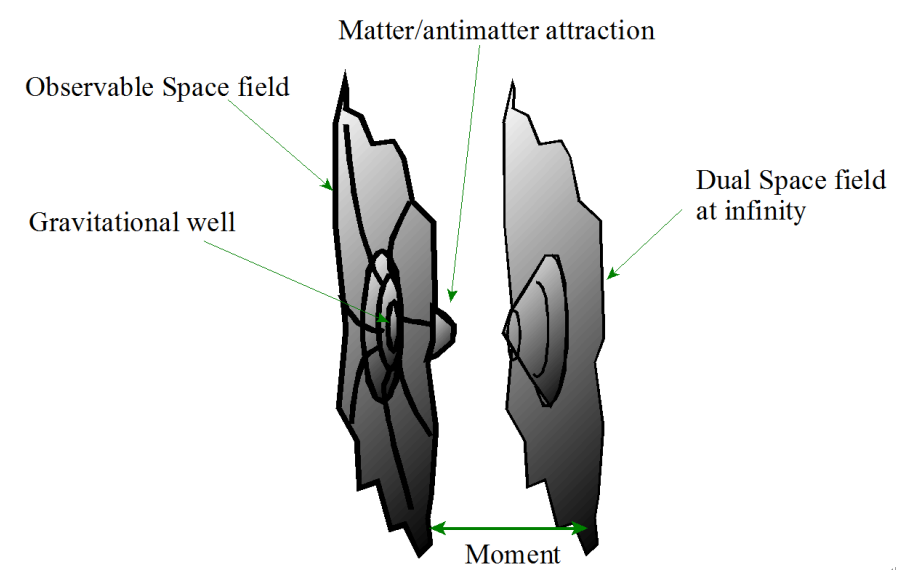

Figure 16. The mirror symmetry of matter and antimatter forming mirror spaces separated by a moment dipole of time. One can image these states like magnetic monopoles ordered in space and time.

- The parallel spacetime membranes interact through mirror symmetry forces that act in a fourth dimension of a time field that represents each moment dipole between the two membranes. The membranes are close together in a moment time field as an ordered set obeying Principle A.

- The classical space field of Matter is a moment in time in the past of the future Antimatter space field. The dipolar space fields interact through force fields I call the White force. Thus, the spaces have curvature due to the interactions between matter and antimatter.

- The contours and curvatures of these mirror spaces determine how a clock beats as a result of the momentdipole gap between two states. This also determines the complementary properties of the states. Thus quantum properties become classical at moment dipole time spans greater than the moment gap.

- Interestingly, quantum properties become dependent on the mass of states and the dipole moment measures, which in turn depend on the curvature of the spaces due to the White field. The moment gap determines how fast one can measure conjugate quantum properties of a given set of paired states.

- Thus the smallest gap between the membranes is Plank time and that determines the formation of blackholes connecting the sheets.

- Note that blackholes will not be singularities. They are donut holes connecting the spaces at infinity!

- The white force between the space membranes is attractive but there is a repulsive maximization field associated with each space. Maximization occurs in the spaces, minimization occurs in the moment gap.

- Like the magnetic dipole field the white force has a maximization field called the dark repulsive field. The dark repulsive field expands the universe. The white force is like the magnetic field. It is a dipole of the dark force.

- The electric field is a monopole field of the dipole magnetic field. The dark field is a 4-dimentional monopole field that wants to expand the space field to infinity just as an electric field is propagated to infinity as a 3-D monopole field.

- The white attractive force attraction between conjugate states induces spacetime curvature between of the parallel conjugate space fields and defines what we call a "moment in time". So one can see why curvature is induced as gravitational fields that relate to the general relativistic picture of a gravitational field.

- The attraction of matter and antimatter dipolar reestablishes the symmetry of the states and also deforms the parallel sheets as gravitational wells.

- In large galaxies, the effects of cross linking between matter and antimatter states across the White field appear as unseen dark forces making galaxies move in peculiar ways.

- The white attractive force will also cause MOND effects and other effects that we see in nature. There appears to be more missing mass that is stored in the White field, since the White field interacts with matter and unseen antimatter!

- The white force is a very strong force since the more matter you add on the space membrane, the more interactions you get between the conjugate states. 
As an example, if one considers a proton on one sheet and an antiproton on the other sheet as shown in Figure 17, one gets a certain attraction that deforms the space fields to give the mass of the proton as a gravitational well. If one adds more Protons close together, such as 2 more, one gets a total of 4 attractive interactions between the conjugate states that can overcome the repulsion of the electric field. So to overcome the repulsion of the electric fields, one must add neutrons between the protons to get a total of 9 attractive interactions holding them together in each space field, while one gets only 2 repulsions for each pair in each space field. As shown in Figure 18, the attractive force between the protons and neutrons is exponentially related as a strong force to their numbers.

One can see where the strong and the gravitational forces come from! One can imagine the two parallel universes as a way to time-ordering conjugate information so that they are infinitely spatially apart and yet ordered as future-past possibilities. There is no contradiction of information states in a logical continuum. Since the two spaces are infinitely far apart no one observer could observe an illogical universe, and the states are also ordered in time as past-future so that they do occur at the same instant. You can think of Matter and Antimatter as two time-ordered states separated by a logical causality so that they cannot interact. When they do, they annihilate each other to zero information states.

Each moment the mind or observer lifts a space field from a dipole moment field and establishes a monopole Minkowski Past Space field as a result. When at one moment one measures an electron as having spin-up in such a sheet, the moment dipole is lifted as a causally consistent monopole universe as a result that makes that state causally consistent from the Big Bang to the here-now. One can lift a new moment dipole into a new mo-

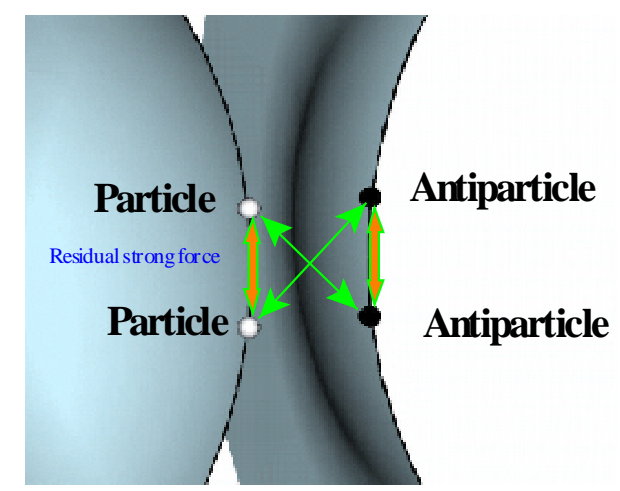

Figure 17. Deformations of the space sheets result in gravitational fields as side effects in space due to the attraction of the matter-antimatter states through the White fields. These multiple attractions lead to stronger fields such as the Yakuwa potential between quarks.

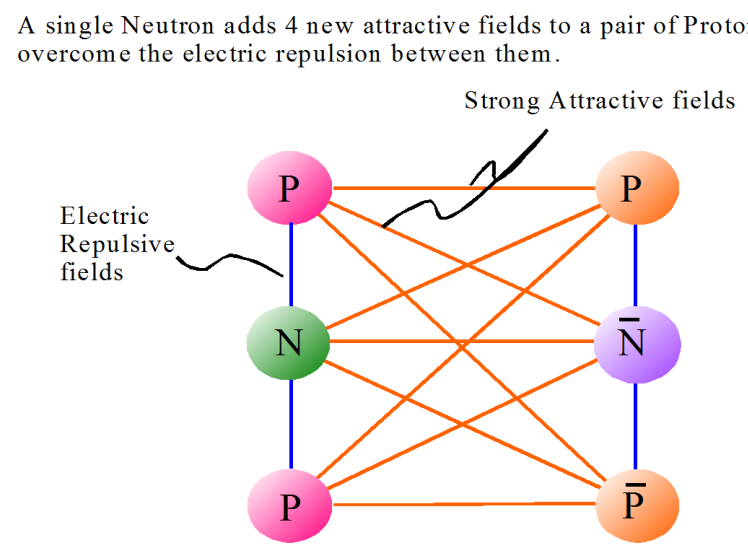

Figure 18. The attraction of protons and neutrons can be understood if one considers the symmetry between matter and antimatter and the multipolar forces attracting them even when the electric field is repulsive in the spacetime continuum. 
nopole past space field and observe a spin-down that is also found to be consistent with that moment of observation throughout the space field. So each moment, a universe is created that has a consistent structure with a memory of the previous one (causality).

The concept of maximization could be extended to result in fractional space maximization by maximizing states in only some preferred dimensions. The maximization process that results in fractional maximization of the Space field in only some dimensions of Space can result in space strings and space membranes of illogical pieces of Space with fractional White, Dark, Electric and Magnetic charges. We call such states Quarks. This concept has a rigid basis on a logical continuum.

- A quantum observation results in a logical classical monopole charge field as an outcome in a maximal space field. One can consider such quantum charges as logical truth charges expressed in a classical logical continuum.

- An electric charge is just another logical quantum charge whose conjugate antimatter state is taken to infinity.

- A logical quantum charge that is not fully lifted as maximized probability charge cannot form a logical statement in all dimensions. It forms partial logical charges in the logical space field. One can model quark electric charges as such.

- When such a partial quantum state is lifted in only some of the available spatial dimensions and not in all, one gets fractional quark charges. These charges reflect the illogical states of the un-lifted dimensions since a logical state must fill a logical space field as statement of a result of observation.

- Quarks are illogical states and one cannot observe them as singular complete logical charges in a logical classical space field. These quark states and leptons can be understood by the decomposition of a 3-D space using mathematics.

The Thurston decompositions of 3-D space result in states shown in Table 1.

For example the decomposition of a Neutron is given by:

$$
\left.\frac{u}{d \times d} \rightarrow\left[\frac{E^{D} \times E^{2 G}}{E^{2 D} \times E^{G} \times E^{2 D} \times E^{G}}\right] \rightarrow\left[\frac{E^{D} \times E^{2 G} \times E^{D} \times E^{2 G}}{E^{2 D} \times E^{G}}\right] \times \underset{\text { proton }}{\left[E^{-3 D}\right]} \underset{\text { anti neutrino }}{\underset{\text { electron }}{-3 G}}\right] .
$$

Table 1. The Thurston decomposition of the spacetime field into fermions and bosons.

\begin{tabular}{|c|c|c|c|c|}
\hline \multirow{4}{*}{$\begin{array}{l}\text { Euclidean electron, positron } u, d \text {, } \\
\text { quarks, and anti-quarks electron, } \\
\text { neutrino, and anti-neutrino. }\end{array}$} & Electrons positrons & \multicolumn{3}{|c|}{$E^{3 G}$} \\
\hline & Quarks (+2/3e) anti-Quarks & $E^{D} \times E^{2 \mathrm{G}}$ & $E^{D} \times E^{2 \mathrm{G}}$ & $E^{D} \times E^{2 G}$ \\
\hline & Quarks (+1/3e) & $E^{2 D} \times E^{\mathrm{G}}$ & $E^{2 D} \times E^{\mathrm{G}}$ & $E^{2 D} \times E^{\mathrm{G}}$ \\
\hline & $v_{e}$ & \multicolumn{3}{|c|}{$E^{3 D}$} \\
\hline \multirow{4}{*}{$\begin{array}{l}\text { Spherical muon anti-muon } \\
\text { C, S quarks and anti-quarks, } \\
\text { muon, neutrino and } \\
\text { anti-neutrino. }\end{array}$} & (Muon) $\mu^{+}$ & \multicolumn{3}{|c|}{$S^{3 \mathrm{G}}$} \\
\hline & Quarks (+2/3e) & $E^{D} \times S^{2 \mathrm{G}}$ & $E^{D} \times S^{2 G}$ & $E^{D} \times S^{2 G}$ \\
\hline & Quarks (+1/3e) & $E^{G} \times S^{2 \mathrm{D}}$ & $E^{G} \times S^{2 \mathrm{D}}$ & $E^{G} \times S^{2 \mathrm{D}}$ \\
\hline & $v_{\mu}$ & \multicolumn{3}{|c|}{$S^{3 \mathrm{D}}$} \\
\hline \multirow{4}{*}{$\begin{array}{l}\text { Hyperbolic taon, anti-taon, } \\
\mathrm{T} \text { and B quarks and anti-quarks } \\
\text { and the taon, neutrino, } \\
\text { and anti-neutrino. }\end{array}$} & (Taons) $+\tau$ & \multicolumn{3}{|c|}{$H^{3 \mathrm{G}}$} \\
\hline & Quarks (+2/3e) & $E^{D} \times H^{2 G}$ & $E^{D} \times H^{2 \mathrm{G}}$ & $E^{D} \times H^{2 G}$ \\
\hline & Quarks (+1/3e) & $E^{G} \times H^{2 \mathrm{D}}$ & $E^{G} \times H^{2 \mathrm{D}}$ & $E^{G} \times H^{2 \mathrm{D}}$ \\
\hline & $v_{\tau}$ & \multicolumn{3}{|c|}{$H^{3 \mathrm{D}}$} \\
\hline$S L 2$ & $\gamma$ & \multicolumn{3}{|c|}{$S L 2^{(R D)}$} \\
\hline Photon and $\mathrm{Z}^{\circ}$ & $\mathrm{Z}^{0}$ & \multicolumn{3}{|c|}{$S L 2^{(R G)}$} \\
\hline Nil & $\mathrm{W}+\mathrm{W}-$ & \multicolumn{3}{|c|}{$\operatorname{Isom}^{0}\left(\mathrm{Nil}^{\mathrm{G}}\right) \mathrm{R} 1(\pi) \times \operatorname{Isom}^{0}(\mathrm{NilG})$} \\
\hline Sol & 8 gluons & \multicolumn{3}{|c|}{8 connected components } \\
\hline
\end{tabular}


Table 1 shows the electric and magnetic charges obtained from the Thurston decomposition of 3D spaces. Table 2 shows the decompositions by maximization and minimization of the spacetime field due to fractional dimensional maximization of space (electric) and time (magnetic (predicted)). In later papers I will show how this theory can be extended to predict the existence of super magnetic quarks that have fractional magnetic charges just as the quarks have fractional electric charges. The theory explains why we cannot observe quarks as logically consistent states because they are illogical charges that are incomplete in a logical three-dimensional world. The theory shows how all the forces are decomposed from a fundamental White-Dark field that ties the symmetry of matter and antimatter through the moment dipole time field. So we have the White-Dark field, the Space-Time field, the Electro-Magnetic field as primary 3D fields, and then the fractional fields occur between the fractional membranes and Strings as the Strong-Weak fields, and the other quantum properties we see in nature. The strong and weak force looks like fragmented white attractive and fragmented dark repulsive fields. The gravitational field is tangential bleed through of the White field causing space deformations between the conjugate membranes.

In Table 2 the 3-D Thurston decomposition is shown representing the leptons and the fermions found in nature. For example, the electron is regarded as a fully unexpanded field of space field locked in a quantum charge, the electric charge, $e^{ \pm}$. Quark charges follow as decompositions of space in less than 3-dimensions forming two-dimensional membranes and 1-dimensional strings. Magnetic quarks represent decompositions in the timedomain.

As seen in Figure 16, the strange wobbling of galaxies and stars attributed to dark matter is explained by the white forces between unseen antimatter states on a conjugate mirror space sheet interacting with matter states

Table 2. The decomposition of spacetime by maximization and minimization fields.

\begin{tabular}{|c|c|c|c|c|c|c|c|c|c|}
\hline \multirow{2}{*}{$\begin{array}{l}\text { Thurston } \\
\text { decompositions } \\
\text { of 3-D space }\end{array}$} & \multicolumn{4}{|c|}{$\begin{array}{l}\text { Decompositions of the space field by } \\
\text { dark field maximization }\end{array}$} & \multicolumn{5}{|c|}{$\begin{array}{l}\text { Decompositions of the space field by } \\
\text { White field minimization }\end{array}$} \\
\hline & \multicolumn{4}{|c|}{ Leptons } & \multicolumn{4}{|c|}{ Quantum fields } & \\
\hline Maximized dimension & -dimensior & 1-dimension & 2-dimension & 3-dimension & 0-dimension & 2-dimension & 1-dimension & -dimension & \\
\hline Fields & $\begin{array}{l}e^{ \pm 1} \\
\text { Electric } \\
\text { fields }\end{array}$ & $\begin{array}{c}e^{ \pm \frac{2}{3}} \\
\text { Weak fields }\end{array}$ & $\begin{array}{c}e^{ \pm \frac{1}{3}} \\
\text { Weak fields }\end{array}$ & \multirow{4}{*}{$\begin{array}{c}e^{ \pm 0} \\
\text { Space field } \\
\text { monopole }\end{array}$} & \multirow{4}{*}{$\begin{array}{c}h^{ \pm 0} \\
\text { Time field } \\
\text { moment } \\
\text { dipole }\end{array}$} & $\begin{array}{l}h^{+\frac{1}{3}} \\
\text { Color fields }\end{array}$ & $\begin{array}{l}h^{ \pm \frac{2}{3}} \\
\text { Color fields }\end{array}$ & $h^{ \pm 1}$ & \\
\hline Euclidean & $e^{ \pm 1}$ & u-quark & d-quark & & & $\begin{array}{l}\text { Magnetic } \\
\text { u-quark }\end{array}$ & $\begin{array}{c}\text { Spin } \\
\text { charges }\end{array}$ & $\begin{array}{l}\text { Magnetic } \\
\text { fields }\end{array}$ & \\
\hline $\begin{array}{l}\text { Massive } \\
\text { states }\end{array}$ & $\mu^{ \pm 1}$ & c-quark & s-quark & & & $\begin{array}{l}\text { Magnetic } \\
\text { d-quark }\end{array}$ & $\begin{array}{l}\text { Magnetic } \\
\text { s-quark }\end{array}$ & & \\
\hline Hyperbolic & $\tau^{ \pm 1}$ & t-quark & b-quark & & & $\begin{array}{l}\text { Magnetic } \\
\text { t-quark }\end{array}$ & $\begin{array}{l}\text { Magnetic } \\
\text { b-quark }\end{array}$ & & \\
\hline \multicolumn{10}{|c|}{ Gauge Bosons } \\
\hline Photons & $e^{ \pm 1}$ & & & & & & & $h^{ \pm 1}$ & \\
\hline \multicolumn{10}{|c|}{ Vector Bosons } \\
\hline \multirow{4}{*}{ Gluons } & $e^{ \pm 1}$ & & & & & $h^{ \pm \frac{1}{3}}$ & $h^{ \pm \frac{2}{3}}$ & & 2 states \\
\hline & & $e^{ \pm \frac{2}{3}}$ & & & & $h^{ \pm \frac{1}{3}}$ & $h^{ \pm \frac{2}{3}}$ & & 2 states \\
\hline & & & $e^{ \pm \frac{1}{3}}$ & & & $h^{ \pm \frac{1}{3}}$ & $h^{ \pm \frac{2}{3}}$ & & 2 states \\
\hline & & & & $e^{ \pm 0}$ & & $h^{ \pm \frac{1}{3}}$ & $h^{ \pm \frac{2}{3}}$ & & 2 states \\
\hline \multicolumn{10}{|c|}{ Intermediate Vector Bosons } \\
\hline $\mathrm{Z}^{0}$ & & & & $e^{ \pm 0}$ & $h^{ \pm 0}$ & & & & \\
\hline \multirow[t]{2}{*}{$W^{ \pm}$} & $e^{ \pm 1}$ & & & & $h^{ \pm 0}$ & & & & \\
\hline & Space & & & $e^{ \pm 0}$ & $h^{ \pm 0}$ & & Time & & \\
\hline
\end{tabular}


on the past space sheet. Obviously one cannot see what exists a moment into the future, but one can sense the matter states interacting with these states through the White field. One can imagine that antimatter copies of the universe of matter is only a moment away but not simultaneously expressed. It all boils down to a logical world that is modeled by a logical mind and by logical laws of nature, after all our minds can only express logical reality. All the laws we create are modeled by logical structure and anything out of that becomes illogical, so we must keep in the mind the role that mind plays in defining our logical laws and logical structure and as such we must use logical structure side by side with illogical quantum structure to model the world.

\section{Probing the Vacuum-Where Did Mass Come from?}

The gravitational potential results because of the logical structure that is permitted in a classical world between two conjugate states. The more logically consistent a quantum state is, the less mass it has! A massless photon can exist as its conjugate in spacetime. The lifetime of a fundamental particle and its conjugate state also depends on how illogical or logically consistent it is. By logical and illogical I do not mean "literal logic". I mean representations that can be interpreted as unitary states from multipolar states. For example one can deduce that the $\pi^{\circ}$ and its antiparticle have a very short life span and cannot exist for prolonged periods of time as logical statement in a logical spacetime continuum. The more complex a quantum structure is the more the mass it will have and the more annihilation White force it has. The attraction between the two membranes relies on logical distinctiveness of the causal structure of the states. Thus one can imagine that photons always form distinct continuums since no two conjugate photons can occupy the same logical continuum. Photons are massless. Suppose a particle has conjugate quantum states that cannot be separated. Such a state will maximize fields only in conjugate variables that can be separated as distinct truth charges. Thus, for example an electron will maximize an electric charge. However, its magnetic charge conjugate cannot be separated across the White field. It also has properties that are statistical in nature since it could have a spin state that conjugates to a previous measurement. Thus the electron will always have mass. Mass leads to blackholes and blackholes annihilate states.

Before one knows if an answer (a quantum property) to a query is a YES or a NO answer, both answers are tied together as a dipole field by a White field. This field is like a dipole field that holds both answers together in a quantum probability field in independent space fields. The correct answer rears its head only when one disturbs the dipole by a query by looking or by experimentation. In quantum theory the answer will manifest as a collapse to a definite state of the wave function of the state we are inspecting. The same principle applies in this theory. However, the appearance of an answer is due to Maximization of logical fields in a logical continuum that demands that one cannot have contradictions of truths. There now exists a theoretical framework and an epistemology for the collapse of the wave function and special relativity theory as well as the standard theory. One cannot get a logical YES/NO dipole answer at the same instant. Only logically consistent monopole answers are permitted.

- When one gets a logical answer, one has disturbed a dipole mystery as symmetry. The measurement must pull apart a set of oppositely charged dipoles to create a monopole logical truth charge that fills the observation field with its truth-charge.

- This truth charge can be any charge one needs to measure for the state. It takes the dipolar forms of [YESNO], [CAT DEAD-CAT ALIVE], [SPIN-UP-SPIN-DOWN], [POSITIVE-NEGATIVE electric charges], [N$\mathrm{S}$ magnetic charges], and other conjugate physical charges, as well as charges that one does not know about at this point in time.

- All such charges will be referred to as Logical Charges. Some logical charges such as the electron are massive while and some are massless. Massive charges still have illogical incompleteness for a true causal structure and so they are still tied into the vacuum to conjugate states by the White field. They generate mass in the form of curvature of the attracting sheets to form gravitational wells.

- The appearance of a result that is consistently true for all parts of the observation space field makes it true that some monopole field has accompanied the observational field. Every time one has a break in symmetry, fields are generated that accompany this symmetry break. These field looks like monopole fields associated with the truth-field selected as an outcome.

- This truth should not find a contradiction in the entire observation logical space field. It is bounded in this monopole information field by causality and classical realism. The contradiction is removed by time ordering! The contradictions of observation and measurements that are generated in a 3-D world are eliminated back to 
symmetry in a 4-D world!

- This means that one cannot generate any motions that are faster than the rate at which this field can transfer information. No matter how fast one travels to catch up and exit this truth information field to experience its opposite value, one cannot surpass the rate at which this field permeates the space field with a solitary truth charge. If one can catch up with this truth field and surpass its rate of permeation or its information propagation speed in our world, one will be able to have experienced opposite realities at the same time as spooky.

The spacetime field is a logically and causally consistent truth. Causality is the ordering of events in the spacetime field for logical consistency! One only observes a past space field never a future space field.

- Each moment results in the lifting of a monopole logical space field from an illogical quantum dipole moment time field. The relationship between space and time is just like the relationship between a monopole electric field that is observable and dipole magnetic fields that are unobservable.

- Space and electric fields are logical fields that are monopolar with accompanying dipole fields that are inseparable as quantum paired states. One cannot observe both future and past; one cannot observe N and S magnetic poles. This generates a view of a logical spacetime field that is consistent with special relativity theory and quantum theory.

- It is important to understand that when one lifts a state from the quantum vacuum as a logical reality, this state can give information that should not be contradicted. The dictum of axioms and theorems is invoked in a real way. Any conclusions one draws by observing this state will be followed by theorems that manifest the truth of axioms in the observation space. One can call this information field a truth-charge that is just like any other charge in nature. Just like an electric charge, if one brings opposite truth charges near one another, they will attract and annihilate so that there should be no contradictions in an observable logical space field. When one measures an electric charge, one is measuring a truth or axiom of some sort that one has yet to understand.

- If one lifts an electron and a positron from the vacuum, one should imagine that one has lifted an opposing truth-value of some logical axioms that want to annihilate each other and make the space field logical again. So fields accompany states that want to annihilate any contradictions in logical realism. When one creates an electron-positron pair, logical realism can be contradicted with quantum properties the pair carries. That is why such paired states can behave spookily when observed.

- This principle of fields accompanying a result is a principle of symmetry compensation that has far-reaching consequences.

- Associated with symmetry is a complex field of higher dimensional information that results in some mystery before a measurement is made. Symmetry is associated with real and complex valued functions that go hand in hand to describe the full symmetry.

Principle C of Causal Conspiracy: An annihilation field accompanies every creation of an information monopole state.

Let us put things in perspective on the Riemann sphere. A gravitational well can result in a blackhole. A blackhole annihilates information back to the vacuum. Imagine a blackhole with a tip at the North Pole (infinity) as shown in Figure 19. It is connecting to conjugate states at infinity. On the parallel space sheets model, it looks like a coupling of Matter and Antimatter sheets. One can move the blackhole on the sphere like a gravitational well on the surface until it reaches and surrounds infinity and vanishes. One can also connect the blackhole to infinity.

Thus, one could restore the vacuum to symmetry by annihilating the gravitational wells in two ways. The first way is to maximize and move the symmetry breaking matter states to infinity. This is what happens in the expanding universe. The second way is to takes states via a shortcut to infinity by means of a gravitational annihilation sink at infinity that is referred to as a blackhole.

\section{Application of Theory to Quantum Theory}

\subsection{Uncertainty Principle}

The parallel sheets configuration can tell one why there is uncertainty in quantum theory. The relationship be- 
The two faces of Gravity as a Dark repulsive field and as an attractive field. Infinity

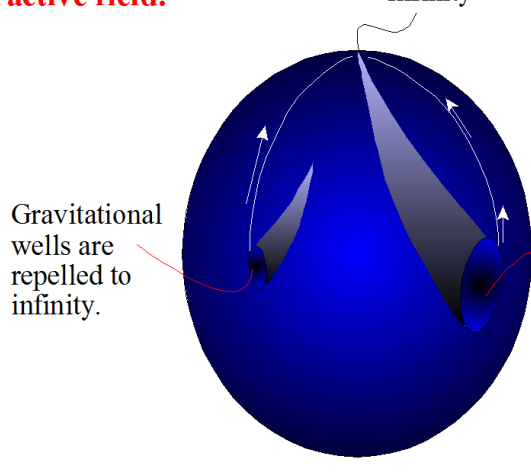

The gravitational well is just the other way information states want to be consistent in a space field.

Black hole connects local states to infinity.

Black holes are being repelled to infinity and that is why galaxies are moving apart.

Figure 19. There are two paths to annihilate information that has created: a) Deform the space and take the states to infinity by creating minimization fields we call gravitational wells that connect to infinity as blackholes; and b) Expand the states by maximization to infinity (expanding universe). Both ways result in the annihilation of information states created after the Big Bang.

tween past present and future gives us a model for causality. This model imposes the condition that the past and the future are derivatives of the present instant action in that when an event occurs the past and future of that event become established simultaneously on parallel spaces. To see how symmetry the Euler-Lagrange equation and the past present and future are related write a general discrete Lagrangian for a particle. Consider dynamical changes from one time frame to another and assuming no mass changes for the two time states. The general time-ordered Lagrangian with identified time and space coordinate variables and for small changes in all the variables is:

$$
L_{q \bar{q}}=\frac{m}{2 \Delta t}\left(\Delta q_{t_{2}}-\Delta q_{t_{1}}\right)^{2}-\frac{\Delta t}{2} V\left(\Delta q_{t_{2}}\right)-\frac{\Delta t}{2} V\left(\Delta q_{t_{1}}\right)
$$

The transitions from the present $(n)$ to the future $(f)$ and from the past $(p)$ with the complex past-potential being of opposite polarity to the complex future potentials represents the CPT symmetry of matter and antimatter states. Assume that it occurs in a small interval of the Time field $\Delta t$. The Feynman path integral for this small interval is given by the conjugate equations from past to present as:

$$
q_{p}|\boldsymbol{T}| q_{n}=\eta_{n \rightarrow p} \mathrm{e}^{\left\{i\left[\frac{m}{2 \Delta t}\left(\Delta q_{n}-i \Delta q_{p}\right)^{2}-\frac{\Delta t}{2} V\left(\Delta q_{p}\right)+\frac{\Delta t}{2} V\left(\Delta q_{n}\right)\right]\right\}}
$$

and for the transition from the present to the future:

$$
q_{n}|\boldsymbol{T}| q_{f}=\eta_{n \rightarrow f} \mathrm{e}^{\left\{\left[\left[\frac{m}{2 \Delta t}\left(\Delta q_{f}-i \Delta q_{n}\right)^{2}-\frac{\Delta t}{2} V\left(\Delta q_{n}\right)-\frac{\Delta t}{2} V\left(\Delta q_{f}\right)\right]\right\}\right.}
$$

and again from future to past as:

$$
q_{f}|\boldsymbol{T}| q_{p}=\eta_{f \rightarrow p} \mathrm{e}^{\left\{\left[\frac{m}{2 \Delta t}\left(\Delta q_{p}-i \Delta q_{f}\right)^{2}+\frac{\Delta t}{2} V\left(\Delta q_{f}\right) \mp \frac{\Delta t}{2} V\left(\Delta q_{p}\right)\right]\right\}}
$$

The transfer matrix for the combined evolution from the now to the past and from the now to the future is given by:

$$
q_{p}|\boldsymbol{T}| q_{n} q_{n}|\boldsymbol{T}| q_{f}=\eta_{p \rightarrow n} \eta_{n \rightarrow f} \mathrm{e}^{\left\{-i\left[\frac{m}{2 \Delta t}\left[\left(\Delta q_{p}-i \Delta q_{f}\right)^{2}+2 i\left(\Delta q_{p} \Delta q_{n}+\Delta q_{n} \Delta q_{f}+\Delta q_{f} \Delta q_{p}\right)\right]+\frac{\Delta t}{2} V\left(\Delta q_{f}\right) \mp \frac{\Delta t}{2} V\left(\Delta q_{p}\right)\right]\right\}}
$$

Finally the evolution becomes a closed spacetime loop when we go back to the past from the future so that:

$$
q_{p}|\boldsymbol{T}| q_{n} q_{n}|\boldsymbol{T}| q_{f} q_{f}|\boldsymbol{T}| q_{p}=\eta_{p \rightarrow n} \eta_{n \rightarrow f} \eta_{f \rightarrow p} \mathrm{e}^{\left\{\left[\frac{m}{\Delta t}\left(\Delta q_{p} \Delta q_{n}+\Delta q_{n} \Delta q_{f}+\Delta q_{f} \Delta q_{p}\right)\right]\right\}}
$$


As $\Delta t \rightarrow 0$ relation (13) becomes a differential relation with anti-commutation relations. The reason for the change in sign to anti-commutators is due to the opposite polarity of the motions since the way time changes for each state depends on the direction being positive or negative. For example as time moves forward the present will be negative with respect to the future and the past will be negative with respect to the present and the future will be positive with respect to the past so that if one uses arrows to represent this:

$$
\begin{aligned}
& q_{p}|\boldsymbol{T}| q_{n} q_{n}|\boldsymbol{T}| q_{f} q_{f}|\boldsymbol{T}| q_{p} \\
& \quad=\eta_{p \rightarrow n} \eta_{n \rightarrow f} \eta_{f \rightarrow p} \exp \left\{m\left\{\left(\Delta q_{n} \frac{\Delta q_{p}}{\overline{\Delta t}}-\Delta q_{f} \frac{\Delta q_{n}}{\overline{\Delta t}}\right)+\left(\Delta q_{p} \frac{\Delta q_{n}}{\overline{\Delta t}}-\Delta q_{n} \frac{\Delta q_{p}}{\overline{\Delta t}}\right)-\left(\Delta q_{f} \frac{\Delta q_{p}}{\overline{\Delta t}}-\Delta q_{p} \frac{\Delta q_{f}}{\overline{\Delta t}}\right)\right\}\right\} .
\end{aligned}
$$

In the presence of the White field and matter, $\Delta t \neq 0$, the total phase change from past to present to future and back to the past must be due to a minimum action allowable in Nature. Label this action $h$. Going around a spacetime loop in which matter is present and if $\Delta t=0$, the minimum action is given by the limiting relationship:

$$
h_{\text {min }}=\lim _{\Delta t \rightarrow 0} h_{\min }=\lim _{\Delta t \rightarrow 0} m\left\{\left(\Delta q_{n} \frac{\Delta q_{p}}{\overline{\Delta t}}-\Delta q_{f} \frac{\Delta q_{n}}{\overline{\Delta t}}\right)+\left(\Delta q_{p} \frac{\Delta q_{n}}{\overline{\Delta t}}-\Delta q_{n} \frac{\Delta q_{p}}{\overline{\Delta t}}\right)-\left(\Delta q_{f} \frac{\Delta q_{p}}{\overline{\Delta t}}-\Delta q_{p} \frac{\Delta q_{f}}{\overline{\Delta t}}\right)\right\} .
$$

Note that the labels that define the actions do not allow a proper action to be defined since the coordinate labels are in different times from the velocity labels. The time-operators cannot act simultaneously on any two time-separated spatial labels. For example a future label $f$, and a now label $n$, cannot be acted upon simultaneously by the time-operator. Thus only pseudo action quantities are present when the labels differ. The labels coincide to a single real variable when $\Delta t \rightarrow 0$. This leads to the anti-commutator relations:

$$
h_{\min }=\lim _{\Delta t \rightarrow 0} h_{\min }=\lim _{\Delta t \rightarrow 0} m\left(\Delta q_{n} \frac{\Delta q_{n}}{\overrightarrow{\Delta t}}-\Delta q_{n} \frac{\Delta q_{n}}{\overline{\Delta t}}\right)=0
$$

However, $\Delta t \neq 0$, and so the limiting action cannot be zero and must have a finite value since the three components must have the same values due to the conservation of momentum, and so the two terms vanish leaving only one term. In the near limit to zero (never zero) one gets a constant action known as Plank's constant:

$$
\frac{i h}{2 \pi}=\lim _{\Delta t \rightarrow \delta t} \frac{i h}{2 \pi}=\lim _{\Delta t \rightarrow \delta t}\left[m\left(\Delta q_{f} \frac{\Delta q_{p}}{\overline{\Delta t}}-\Delta q_{p} \frac{\Delta q_{f}}{\overline{\Delta t}}\right)\right], \quad f=p+\delta t
$$

Equation (17) shows that the action cannot be specified by simultaneously measuring the velocity variable and the position variable in one time label. This is an amazing revelation. The Uncertainty Principle is simply stating that if one makes a measurement and disturbs symmetry; the entire symmetry must go through an infinitesimal spacetime loop to get back to a symmetric state. The final state must always have a different time label in either the coordinate variable or the velocity variable. Thus the continuum must be elevated to a higher dimension to get symmetry again. This is the minimum break in symmetry allowed in Nature and so no two complimentary variables of velocity and position can be measured simultaneously. The finite limit of the "derivative" must be a constant of causality as stated earlier and, $\frac{\Delta q_{j}}{\Delta t}=c$. If this restriction did not apply and there was no future and past representations of a space sheets, there would be no Plank's constant and the limiting velocity of signals in Nature would be infinite. This of course would lead to a world where the moment of experience is instantaneous and certainty and perfect symmetry will rule. In such a perfectly Euclidean world, no break in symmetry can occur. So the presence of a finite and definite limit to the quantum spacetime that defines the causality and it forces a moment separating the past from the future results and also provides for a finite limit to the speed of information and a definite value for Plank's constant. A very important detail is that the time-ordered operators act as directional differentials:

$$
\frac{\partial}{\partial t}\left[q_{n} q_{f}\right]=q_{n} \frac{\partial \vec{q}_{f}}{\partial t}+q_{f} \frac{\partial \bar{q}_{n}}{\partial t}=q_{n} \frac{\partial q_{f}}{\partial t}-q_{f} \frac{\partial q_{n}}{\partial t}
$$


It is therefore apparent that causality applies even at the atomic level and there is no conflict with the uncertainty relations contrary to common statements made in present day theoretical work. In fact the Uncertainty Principle is a consequence of the time-ordered causal behavior of the world. The above findings are to be understood as follows:

Principle of Causality: Every observable monopole state in a maximized space field has symmetry in a dipole-field ordering of past and future and its physical observables comprise complimentary properties that can only act in a causal manner on one state at a time.

This leads us to two important conclusions:

The Principle of Uncertainty: The simultaneous measurement of space and time complimentary variables (such as the position and momentum, energy and time for a moving particle) is limited by time moment ordering in a logical continuum.

This is a direct consequence of a break in CPT symmetry. If CPT symmetry were upheld then the states would appear to be identical and spookiness would prevail above causality.

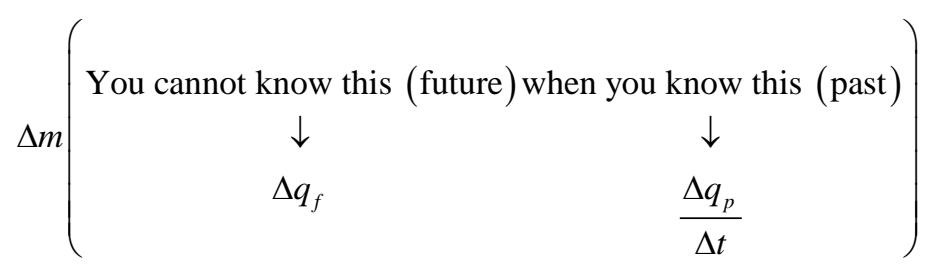

The knowledge of the two states can only be obtained in a maximized Monopole Space-field lifted at the expense of an unmanifested future Space field (its compliment sent to infinity). Such knowledge is associated with a quantity of action due to the unmanifested observables that can only exist if the two states are separated by a moment dipole of time.

\subsection{The Principle of Least Action}

Principle of Least Action: A system moves from one configuration to another in such a way that the variation of the integral $\int L \mathrm{~d} t$ between the path taken and a neighboring virtual path co-terminus in space and time with the actual path is zero.

By considering the time-ordered actions of the Lagrangian evolution we arrive at the Principle of Hamilton albeit in a backwards manner. This is not a rigorous proof but it shows the spirit of the principle as associated with past and future actions. Start with the uncertainty relations (16),

$$
L_{p f} \Delta t=m\left(\Delta q_{f} \frac{\Delta q_{p}}{\Delta t}-\Delta q_{p} \frac{\Delta q_{f}}{\Delta t}\right)=\frac{i h}{2 \pi},
$$

Changing to differentials:

$$
\begin{gathered}
L_{p f} \partial t=m\left(\mathrm{~d} q_{f} \dot{q}_{p}-\mathrm{d} q_{p} \dot{q}_{f}\right)=\frac{i h}{2 \pi}, \\
\partial L_{p f}=m\left(\mathrm{~d} q_{f} \ddot{q}_{p}+\dot{q}_{f} \dot{q}_{p}-\mathrm{d} q_{p} \ddot{q}_{f}-\dot{q}_{p} \dot{q}_{f}\right)=\frac{i h}{2 \pi} \frac{\partial}{\partial t},
\end{gathered}
$$

Differentiating with respect to $\dot{q}_{p}$, 


$$
\frac{\partial L_{p f}}{\partial \dot{q}_{p}}=m\left(\mathrm{~d} q_{f} \frac{\partial\left(\ddot{q}_{p}\right)}{\partial \dot{q}_{p}}-\partial t \ddot{q}_{f}\right)=0,
$$

Now $\mathrm{d} t \rightarrow 0$ so and we see that the past terms have completely disappeared from the right hand side and the terms are certain instead of uncertain i.e. true physical variables:

$$
\frac{\partial L_{p f}}{\partial \dot{q}_{p}}=\frac{\partial}{\partial \dot{q}_{f}}\left(m \mathrm{~d} q_{f} \frac{\partial\left(\dot{q}_{f}\right)}{\partial t}\right)=0
$$

Note that the relationship has right-hand terms that are pure actions with the same time labels. The mixture of terms has been eliminated. Differentiating with respect to time,

$$
\frac{\mathrm{d}}{\mathrm{d} t}\left(\frac{\partial L_{p f}}{\partial \dot{q}_{p}}\right)-\frac{\partial}{\partial \dot{q}_{f}}\left(m \frac{\mathrm{d} q_{f}}{\mathrm{~d} t} \cdot \frac{\mathrm{d} q_{f}}{\mathrm{~d} t}+m \frac{\mathrm{d}^{2} q_{f}}{\mathrm{~d} t^{2}} \mathrm{~d} q_{f}\right)=0
$$

Putting $E_{f}=m \frac{\mathrm{d} q_{f}}{\mathrm{~d} t} \cdot \frac{\mathrm{d} q_{f}}{\mathrm{~d} t}$, for the kinematic energy and, $V_{p}=-m \frac{\mathrm{d}^{2} q_{f}}{\mathrm{~d} t^{2}} \mathrm{~d} q_{f}$, for the potential energy:

$$
\begin{gathered}
\frac{\mathrm{d}}{\mathrm{d} t}\left(\frac{\partial L_{p f}}{\partial \dot{q}_{p}}\right)-\frac{\partial}{\partial \dot{q}_{f}}\left(E_{f}-V_{f}\right)=0 \\
\frac{\mathrm{d}}{\mathrm{d} t}\left(\frac{\partial L_{p f}}{\partial \dot{q}_{p}}\right)-\frac{\partial L_{f}}{\partial q_{f}}=0
\end{gathered}
$$

It is important to note that the velocity and position variable still retain their respective time labels enforcing uncertainty even in this seemingly classical system. What this means is that when we take the Lagrangian, $L_{p f}$, of the complete set of symmetry states there is uncertainty since the terms are given by a mixture of states and Nature cannot allow arbitrary paths with unphysical variables with different time labels. These unphysical actions are not acceptable. The only way that Nature has found to eliminate this uncertainty and allow a real energy term action on the right hand side of the Euler-Lagrange equation and a trajectory that still allows the velocity and the position variables to be at different time labels:

$$
\frac{\mathrm{d}}{\mathrm{d} t}\left(\frac{\partial L_{p f}}{\partial \dot{q}_{p}}\right)=\frac{\partial L_{f}}{\partial q_{f}}
$$

Thus the velocity terms annihilate one another so that only future or past terms result for the path selected by Nature. The path selected by Nature still retains its uncertainty but the symmetry takes a maximum between past and future. There are six such equations that govern the motion simultaneously forming two closed loops and these can be obtained by an exchange of labels:

$$
\begin{aligned}
& \frac{\mathrm{d}}{\mathrm{d} t}\left(\frac{\partial L_{p n}}{\partial \dot{\boldsymbol{q}}_{p}}\right)=\frac{\partial L_{n}}{\partial q_{n}}, \quad \frac{\mathrm{d}}{\mathrm{d} t}\left(\frac{\partial L_{p f}}{\partial \dot{\boldsymbol{q}}_{n}}\right)=\frac{\partial L_{p}}{\partial q_{p}}, \\
& \left.\frac{\mathrm{d}}{\mathrm{d} t}\left(\frac{\partial L_{p f}}{\partial \dot{q}_{n}}\right)=\frac{\partial L_{f}}{\partial q_{f}}, \quad \frac{\mathrm{d}}{\mathrm{d} t}\left(\frac{\partial L_{p f}}{\partial \dot{q}_{f}}\right)=\frac{\partial L_{n}}{\partial q_{n}},\right\} \\
& \frac{\mathrm{d}}{\mathrm{d} t}\left(\frac{\partial L_{p f}}{\partial \dot{\boldsymbol{q}}_{f}}\right)=\frac{\partial L_{p}}{\partial q_{p}}, \quad \frac{\mathrm{d}}{\mathrm{d} t}\left(\frac{\partial L_{p f}}{\partial \dot{q}_{p}}\right)=\frac{\partial L_{f}}{\partial q_{f}},
\end{aligned}
$$

We find that Nature cannot possibly offer an arbitrary path for motion since Nature itself has no way of calculating the momentum and the position simultaneously. I here state a fundamental immutable fact of experience: 
Statement of Symmetry: A system moves from one configuration to another in such a way that the variation of the integral $\int L \mathrm{~d} t$, between the actual path taken and a neighboring virtual path co-terminus in space and time with the actual path maximizes spacetime symmetry in the Lagrangian.

$$
\left.\begin{array}{c}
\text { Uncertainty } \rightarrow \text { Certainty } \\
\frac{\mathrm{d}}{\mathrm{d} t}\left(\frac{\partial L_{p f \rightarrow p}}{\partial \dot{\boldsymbol{q}}_{p}}\right)=\frac{\partial L_{f \rightarrow p}}{\partial \boldsymbol{q}_{f \rightarrow p}} \rightarrow \frac{\mathrm{d}}{\mathrm{d} t}\left(\frac{\partial L_{p}}{\partial \dot{q}_{p}}\right)=\frac{\partial L_{p}}{\partial q_{p}},
\end{array}\right\}
$$

\subsection{Special Theory of Relativity}

Special relativity can be obtained from quantum theory and the concepts of Möbius transformations of a dipole set to a monopole set when one considers the exchange of information between two observers. The information between two relative states in motion can be represented as a reflection of states in parallel mirror worldliness (one dimensional case) with even and odd images as shown in Figure 20.

There are many ways in which a perfect reflector can be made be made to exhibit the behavior of an imperfect reflector. For example if the reflector walls are normal to the trajectory of an information wave then the intensity of the wave as it is reflected from the walls will depend on the angle between the walls which in turn depends on the matter/antimatter content of the walls. The curvature (De Broglie) causes the worldline of the object to tilt. Thus the intensity of the images on the reflector wall will be reduced by the cosine of the angle made by the worldline of the reflected waves in the Euclidean representation. The intensity of the images seen on the moving frame will be reduced by the factor $\xi$, where,

$$
r^{2}=1-\frac{v^{2}}{c^{2}}=1-\xi^{2}
$$

and $r$, is the reflectivity coefficient of the trap, $v$ is the velocity of the observer relative to the observed, $c$ is the speed of the wave front and $\xi$, is the transmission coefficient of the cage wall. As an example one can imagine $\psi$ as a series of movie frames hitting the worldlines. The movie will appear less intense by a factor $\xi$ as it flashes on each wall and it will also be reflected to the other wall where it will also be observed to be less intense by a factor $\xi^{2}$. This less intense image is also re-reflected to the first wall and will be observed to be even lesser in intensity by a factor $\xi^{3}$ and so on. Here $\xi$ is in general a complex number that may also have a real value when the complex part is zero. This loss of intensity of information is due to the tilting of the walls and results in a series of reflections that follow a specific geometric pattern depending on the geometry of the walls. The series for the consecutive reflected wave-terms for the $k^{\text {th }}$ reflection for a wave with initial intensity $A_{k}$ is given by:

$$
\psi_{k}=A_{k}(i \xi)^{k} \mathrm{e}^{\frac{i k \eta}{2}}
$$

where, $\mathrm{e}^{\frac{i k \eta}{2}}$ is the phase difference due to the path difference $\eta=\frac{4 \pi i l \cos \phi}{\lambda}, l$, is the distance separating the observer worldliness, and $\lambda$ is the wavelength of the wave with $\xi$ a real number. The equation represents the $k^{\text {th }}$ image of the reflected wave components. In quantum theory the time dependent Schrodinger equation can be written as a function of the coordinate function $q_{j}$ and time.

$$
\sum_{j=0}^{\infty}(j)\left[\frac{-h^{2}}{2 m_{j}} \frac{\partial^{2} \Psi}{\partial q^{2}}\right]+V\left(q_{j} \Psi\right)=i h \frac{\partial \Psi}{\partial t}
$$

One finds that the general solution to (33) is given by:

$$
\psi_{k}=A_{k} U_{k} \mathrm{e}^{\frac{i E_{k} t}{h}}
$$

The array of images of $\psi$, represent the distribution of the wave function and its complex conjugate in the 


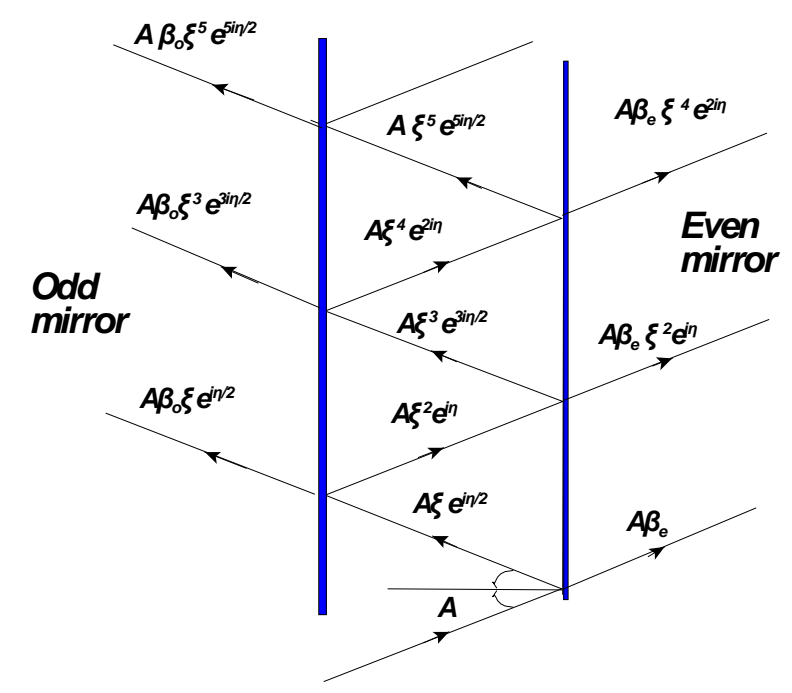

Figure 20. Shows two conjugate quantum waves between two cage walls. If the cage walls are linear one can assume that the reflectivity of the two walls are the same.

past and future of the observer respectively. This is exactly equivalent to the quantum mechanical picture of the probability distribution wave-function and the path integral over all time before a measurement is made. This is evident if one puts $l=i c t, \lambda=\frac{k h}{2 \pi M c}$, and $U_{k}=(i \xi)^{k}$ in Equation (34) and finds that indeed the worldline separation $\lambda$ is caused by the energy content $E$. Note that the time variable $t$ must be transformed to a complex variable for a Euclidean space so that the length variable becomes real. The above relations again point to the transition of time from the Minkowski Spacetime with an indefinite metric to the Euclidean Spacetime with a definite periodic metric. The above relations clearly show that the wave functions conforms to the tenets of the Principles of Causal Conspiracy and quantum theory but without the probabilistic interpretation.

The new interpretation of the Schrödinger equation is that it represents the distribution of the complex representations of the information-wave functions in past and future image spaces $M^{+}$and $M^{-}$. In other words the Schrödinger wave equation is simply the wave equation that describes the intensity of the $M^{+}$and $M^{-}$ images generated by a Casimir information trap and it reflections. When a measurement is made the Casimir trap formed by the worldliness splits the information density (the absolute value of the wave states) into two image components $M^{+}$and $M^{-}$causing a memory of the result to be propagated as conjugate waves of a past event and future possibilities. In parallel mirrors one sees a series of alternating odd and even images. That same thing happens here. The measurement is made by observing information from only the past wall (what is seen just after the measurement). One cannot look at both walls. Thus the collapse of the wave function is when one looks at the past wall and sees only odd and even images in place of complex conjugates. If you look at parallel mirrors, you see an infinite number of images that form a history of the space between the mirrors. These images alternate as left-handed and right-handed images. It corresponds to the transformation:

$$
\psi_{m} \psi_{n} \rightarrow \psi_{\text {odd }} \psi_{\text {even }}
$$

Note that when one says future experiences one means all future iterations from the present, which are just conjugate images of the past. The irony is that the potentiality (conjugation mix) disappears when the state is observed and the definiteness of classical behavior arises simply because the complex wave conjugates propagate as odd and even complex image memories of the result and they cannot be altered by the observer. In quantum theory the probability density is given by:

$$
\psi_{m} \overline{\psi_{n}}=\sum_{m=0}^{m=\infty} \sum_{n=0}^{n=\infty}\left(A_{m} \overline{A_{n}} U_{m} \overline{U_{n}} \mathrm{e}^{\frac{2 \pi i\left(E_{m}-E_{n}\right) t}{h}}\right)
$$

One sees that the expression (36) contains terms of kinds for $m \neq n$ and $m=n$. The states for which 
$m=n$ are independent of time (spacially dependent) since they represent the conjugate states of the wave functions in the Casimir trap made by in the one-dimensional case by the pair of worldlines. While a wave is in transition from one reflector to another, its conjugate does not change with respect to itself in time but its intensity is changing according to the effects of the reflectors $\mathrm{M}^{+}$and $\mathrm{M}^{-}$. Later one shall find that if the topology of the worldliness or reflector walls is such that the reflected images overlap then one can indeed find mixtures of states from two spacetime intervals and some weird effects can be predicted. This would be the case when a particle is moving in some potential wells where its energy exceeds the potential energy it should have during its motion. Instead of probability densities one shall use the term "information-wave density".

Thus the description using the complex conjugate states of spacetime $M^{+}$and anti-spacetime $M^{-}$is equivalent to the splitting of the result into observable odd and observable even states respectively as real past images in complex time. One could write the expectation for the measurement in the split form:

$$
\frac{B_{m} \overline{B_{m}}=A_{e}^{2} \sum_{j=0}^{j=\infty} \varphi_{j} \overline{\varphi_{j}}+A_{0}^{2} \sum_{k=0}^{k=\infty} \varphi_{k} \overline{\varphi_{k}}}{\sum_{m=0}^{m=\infty} \varphi_{m} \overline{\varphi_{m}}}
$$

here, $j$ and $k$ represent even and odd states for all the range of $m$. The observer could also look through the other wall and because of symmetry the amplitude constants $A_{j}$ and $A_{k}$ of the future and past components can be interchanged so that one must have for the mixed terms:

$$
B_{m} \overline{B_{m}}=\frac{\left(A_{e} \sqrt{\sum_{j=2,4}^{\infty} U_{j}^{2}}+i A_{0} \sqrt{\sum_{k=1,3,5}^{\infty} U_{k}^{2}}\right)\left(A_{e} \sqrt{\sum_{j=2,4 \ldots}^{\infty} U_{j}^{2}}-i A_{0} \sqrt{\sum_{k=1,3,5}^{\infty} U_{k}^{2}}\right)}{\sqrt{\sum_{j=1,2,3 \ldots}^{\infty} U_{m}^{2}} \sqrt{\sum_{j=1,2,3 \ldots}^{\infty} U_{m}^{2}}}
$$

In order to see the full beauty of the connection of quantum theory, General and Special Relativity theories with causal conspiracy theory, it is important that we revisit the general relation:

$$
\sum_{m=0}^{\infty} B_{m} \varphi_{m} \bar{B}_{m} \bar{\varphi}_{m}=\sum_{\text {odd }=0}^{\infty} A_{o} \varphi_{\mathrm{ko}} \bar{A}_{o} \bar{\varphi}_{o}+\sum_{\text {even }=0}^{\infty} A_{e} \varphi_{e} \bar{A}_{e} \bar{\varphi}_{e}
$$

One takes the general density wave function:

$$
\varphi_{m} \bar{\varphi}_{m}=\left(f_{e}(\xi)\right)^{2}+\left(f_{o}(\xi)\right)^{2}
$$

This equation represents the resultant intensity of the reflections in even and odd images respectively. But this equation is not quite true. In the above analysis we have separated the odd and even parts of the wave by a "trick" of algebra. This trick is strictly at odds with the reality of the information trap. The images formed by the trap are created by each mirror operating on the wave function and the conjugate wave function such that a progression of operations by each mirror acting on the waves results in odd/even representations in the observed mirror. This can be seen if one follows the wave function as it bounces back and forth between the two trap-walls. If one takes the first trap-wall (A) as having an operator $\alpha$ on the wave and then the second trap-wall (B) as having an operator $\beta$ on the wave then when the wave bounces back and forth one expects the series of intensities to take the form:

$$
\varphi(1-\alpha+\beta \alpha-\alpha \beta \alpha+\alpha \beta \alpha \beta+\cdots)=\varphi\left(1+\sum_{n=0}^{\infty} \beta^{n+1} \alpha^{n} \xi^{2 n+1}(\alpha \xi-1)\right)
$$

The conjugate wave will follow the sequence:

$$
\bar{\varphi}(1-\beta+\alpha \beta-\beta \alpha \beta+\beta \alpha \beta \alpha+\cdots)=\bar{\varphi}\left(1+\sum_{n=0}^{\infty} \alpha^{n+1} \beta^{n} \xi^{2 n+1}(\beta \xi-1)\right)
$$

We see that these cannot be separated out into two separate operators acting on the even and odd numbered reflections respectively. What we want is to have reflections waves that have $\alpha$ and $\beta$ amplitudes for the even and odd images respectively. This means that we want the mirror operators for the wave function to be anti-symmetric. If it were symmetric then both trap walls would affect both the odd and even functions in the same way. Thus when one makes an observation the anti-symmetric wave functions will look the same in any one mirror (exchange of identity of antiparticle and double exchange of identity for the particle). When we impose these conditions one finds that the operators, $\alpha$, or $\beta$, are none other than the Pauli matrices. This can be seen 
if one puts:

$$
0=\psi^{2}-\left(f_{e}(\xi)\right)^{2}-\left(f_{o}(\xi)\right)^{2}=\left[\psi-\alpha f_{e}(\xi)-\beta f_{o}(\xi)\right]\left[\psi+\alpha f_{e}(\xi)+\beta f_{o}(\xi)\right]
$$

and so,

$$
\left.\begin{array}{c}
\alpha_{k}^{2}=1 \\
\beta_{k}^{2}=1 \\
\alpha_{j} \alpha_{k}+\alpha_{k} \alpha_{j}=0 \\
\alpha_{j} \beta_{k}+\beta_{k} \alpha_{j}=0
\end{array}\right\}
$$

Relations (44) impose certain condition on the transformations of the wave functions from an unobserved state in the left-hand side to the observed states of odd and even in the right-hand side. The matrices satisfying these operations are none other than the Pauli matrices. A clear understanding arises naturally since the Exclusion Principle is at work separating odd and even states for anti-symmetric wave functions. The interpretation is that the future and past states cannot commute and so the spacetime information trap walls do not act on themselves as ordinary mirrors do. A frosty mirror will influence all reflections and images it generates of its parallel counterpart. Instead the spacetime mirrors recognize the wave and the conjugate wave separately and uniquely as past and future waves that preserve their separate forms.

We can ask the question "why is it that the Exclusion Principle does not work for material objects in the same moment but only acts in the same space?" This question is profound and fundamental. It separates space from time. The quantum theory generally talks about the Exclusion Principle exclusively for space. It never talks about an Exclusion Principle for time. That two particles of the same genre can live concurrently at the same time but in two different places is clear from experience. Thus time does not have an Exclusion Principle. This difference between space and time becomes evident when we note that an observation of the image complex space shows that the particle-antiparticle are identical when one looks at the past wall due to the transformations of the two space-time walls. Thus even though one sees the same state being propagated into the past the actual states are anti-symmetric wave states. The mapping of motion generated by the theory results in solutions:

$$
\left.\begin{array}{l}
B_{m} \overline{B_{m}}=\frac{\alpha^{2} A_{e}^{2} \sum_{j=\mathrm{even}}^{\infty} \varphi_{j} \overline{\varphi_{k}}+\alpha^{2} A_{o}^{2} \sum_{j=\text { odd }}^{\infty} \varphi_{j} \overline{\varphi_{k}}}{\sum_{m=0}^{\infty} \varphi_{m} \overline{\varphi_{m}}} \\
\overline{B_{m}} B_{m}=\frac{\alpha^{2} A_{e}^{2} \sum_{j=\text { odd }}^{\infty} \varphi_{j} \overline{\varphi_{k}}+\alpha^{2} A_{o}^{2} \sum_{j=\mathrm{even}}^{\infty} \varphi_{j} \overline{\varphi_{k}}}{\sum_{m=0}^{\infty} \varphi_{m} \overline{\varphi_{m}}}
\end{array}\right]
$$

This remains the same as (38) with the Lorentz transform modified for fermions and bosons as follows:

$$
B=\frac{\alpha A_{e}+i \beta A_{o} \xi}{\sqrt{1-\xi^{2}}}, \quad \bar{B}=\frac{\alpha A_{o}+i \beta A_{e} \xi}{\sqrt{1-\xi^{2}}}
$$

If one assumes that a length $X$ is being measured and put in the Euclidean time, $t=i t, A_{e}=x, A_{o}=i c t$, $B=X$, and $\bar{B}=i c T, \xi=\frac{v}{c}$ we get the modified Lorentz transforms:

$$
X=\frac{\alpha x-\beta v t}{\sqrt{1-\left(\frac{v}{c}\right)^{2}}}, \quad T=\frac{\alpha t+\beta \frac{x v}{c^{2}}}{\sqrt{1-\left(\frac{v}{c}\right)^{2}}}
$$

Note that the theory does not restrict the maximum velocity of the states to the speed of light. The parameter $\xi$ can represent the relative velocity of the observers with respect to the maximum maximization speed of information. In fact the theory shows that we could use fermions as information sources if the Pauli matrices are taken into relativistic consideration. Thus if Neutrinos are used, then their information capability undergoes transformations that conform to the form Equation (47) and their velocity does not need to be restricted to light 
speed! Bosons are symmetric in time and fermions are anti-symmetric. From this one concludes that a positron traveling at high speeds relative to an observer will appear to have an internal clock that is running faster than the observer's contrary to what will be expected for an electron with the same motion. This effect may be the reason why the decay rates of Kaons and anti-Kaons seem to be different. The general case for observers in motion with varying energy such as when they are in accelerated frames can be written locally as reflections of a wedge, since a wedge would indicate that the moment field is changing with historic time. The equation for the reflections between the two angled trap walls can be written as:

$$
\psi_{n}=\sum_{n=0}^{\infty} A_{n} \xi^{n} \cos n \theta
$$

The total sum of all the contributions of the normal components of the wave from both mirrors is given by:

$$
B=\frac{A_{e}+i \xi A_{o} \cos \theta}{\sqrt{1-\xi^{2}}},
$$

If $A_{e}=i A_{0}$, then,

$$
B=A_{e} \frac{1-i \xi \cos \theta}{\sqrt{1-\xi^{2}}},
$$

This is the Doppler relativistic formula if one substitutes into Equation (50),

$$
A_{e}=f, B=f^{\prime} \text {, and } \xi=\frac{v}{c} \text {. }
$$

\subsection{Black-Body Radiation}

Thus far one has only considered the one and two-dimensional cases where the one-dimensional case is broken up into odd and even reflection while treating the reflection functions as constants. The cases considered above all involve reflections of the wave function on regular linear traps such as would be experienced when two massless observers in uniform motion measure a parameter. If one extends the concept to the simplest four-dimensional cases involving a time transition then one can conceive of the mirrors as three orthogonal surfaces with the time mirror being orthogonal to the three space mirrors. Thus the waves will be trapped in a four dimensional cavity. The three dimensional cavity is shown in Figure 21 with time being the fourth dimension. If one takes only the time transition of such images then one expects to calculate the evolution of each particle state from the present to the future and from the present to the past. The images of a single particle placed between the mirrors thus formed must give the complete statistical behavior of particles of all types. Such a system is found to be an energy trap such as a black body radiator. In general such systems describe mixtures of particles such as photons and electrons that are both distinguishable and indistinguishable and which conform to the statistics of the Boltzmann Bose and Fermi distributions. If an observer is measuring the frequency of an outgoing wave from a black body radiator then the axis of motion of the wave is that axis which holds no second reflector to stop the ray from escaping the cavity. This is the unique case for Equation (50) when $\theta=\pi / 2$. If one considers the source of waves from particles whose images appear in the mirrors then one has to work out the way the images will appear in the four trap walls. Consider the array of images that result if a single particle is positioned at a fixed distance from three orthogonal mirrors. When an object is placed in the space between say the $y$-z orthogonal mirrors, one sees that the object is reflected many fold back unto itself around the $x-y-z$ mirrors. If one keeps following the progression of reflected images one can get an infinite number of images super-imposed on the object itself. From any object or image one can follow a cyclic progression of images back to the original object through an angle of $2 \pi$. There will be a total of eight (8) object-images each time one rotates through all the mirrors because the $y$ - $z$ mirrors will reflect each other orthogonally and create four images which will be doubled by the $x$ mirror as shown in the Figure 21 .

After each cycle the reflection parameter has acted on all three axes. When we rotate this array of images through the time axis we find that the 8 images will be doubled to 16 images, which because of superposition can indeed become a four dimensional infinite array of images with odd and even states respectively. Suppose the reflection parameters $\xi_{j}$ are not constants it is quite possible to have an array of different object/images supe- 


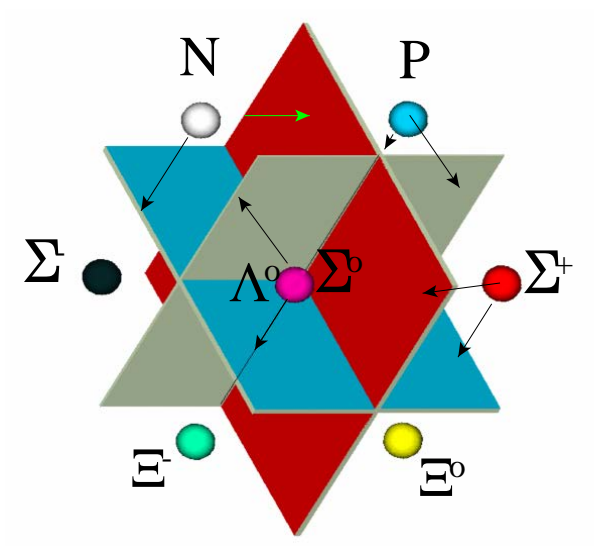

Figure 21. The 8-fold way can be viewed as a triplet of orthogonal mirrors reflecting a single state into 7 images. These can be viewed as a Charge Mirror, a Mass or spin mirror, and Charge-Mass mirror. $(C+M)^{2}=C^{2}+2 C M+M^{2}$.

rimposed on one another in trap Space-time. This is equivalent to stating that there exists forces or acceleration vectors in the space where the object is found since the velocity variables $\xi_{j}$ will not be constant. This opens up the door to a quantum theory of gravity with a definite supporting epistemology based on this theory. Alternatively one could conceive of a gravitational field pulling on the object in some preferred direction of spacetime. The reflections in the "time" axis should also be considered as significant. Here the transformation or reflection parameter is imaginary and no images can exist other than the fact that $x-y-z$ images will go through a cyclic duplication of the first cycle in complex Euclidean time. One must recognize that a transition from the present to the past or future requires the laws of thermodynamics to hold true. Thus the thermal energy will be affected by the reflection parameters through the time dimension. The rotating images in $x-y-z$ space may be what manifest as "spin" and angular momentum. It is as though the images are rotating about the three mirrors $A$ $B$ and $C$ with respect to time in a discrete manner. The rotation parameter in time may be responsible for what one refers to as a direction in the arrow of time since the laws of thermodynamics are uniquely specified to thermal energy loss as time progresses forward and thermal energy gain as time progresses backward. If the space is symmetric the $n^{\text {th }}$ spatial rotation gives the array of images:

$$
A \sum_{n=0}^{\bar{n}} \xi^{n}
$$

and the $m^{\text {th }}$ time rotation thus gives:

$$
A \xi^{n} \sum_{n=0}^{-} \xi^{n}
$$

There could also be permutations of these states by interchange of each image with the particle. Turn attention to the information density wave function. The information wave function is a function of the Space-time variables. In this case the wave function one wants to construct is time-dependent and so is not simply a function of the space variables. In order to construct the wave-function as a function of the variables $\xi_{x}, \xi_{y}, \xi_{z}$ and $\xi_{t}$ note that these variables can be written as functions of the frequency, $v_{j}$ and wavelength, $\lambda_{j}$ of the waves, $\xi_{j}=\frac{u_{j}}{c}=\frac{v_{j} \lambda_{j}}{c}$.

Here $v_{j}$ is the frequency of the wave measured for the $j^{\text {th }}$ wave. The wavelength dependent normalized information density could be transformed to $\xi_{x}, \xi_{y}$ and $\xi_{z}$, variables. The variable $\xi_{t}$, has to do with the way thermal energy is transported through time.

The quantum probability function

$$
\iiint \int \phi \bar{\phi} \mathrm{d} \lambda_{x} \mathrm{~d} \lambda_{y} \mathrm{~d} \lambda_{z} \mathrm{~d} t=1
$$

Is the analogue of the information density function: 


$$
\iiint \int \phi \bar{\phi} \mathrm{d} \xi_{x} \mathrm{~d} \xi_{y} \mathrm{~d} \xi_{z} \mathrm{~d} \xi_{t}=1
$$

Assuming that an infinitesimal loss of energy- $\mathrm{d} E$ occurs as one moves from one moment to the future, and that the future state has only a thermal signature, the $k^{\text {th }}$ spatial information wave function and its conjugate in time will be given by:

$$
\psi_{l, m, n}^{k} \bar{\psi}_{l, m, n}^{k}=\sum_{l=0}^{\infty} \sum_{m=0}^{\infty} \sum_{n=0}^{\infty} \bar{A}_{l, m, n}^{k} A_{l, m, n}^{k} U_{l, m, n}^{k} \bar{U}_{l, m, n}^{k} \mathrm{e}^{\frac{\mathrm{d} E}{\hbar}} \text { (Time Transition rate) }
$$

where the time variable is again complex and the spacetime is Euclidean. Since the reflections in the spatial mirrors are cyclic, the indices $l, m$, and $n$ can be integrated into just a single variable $n$. We note that at any moment, from a single particle with energy $E=h f$ we get a total of $n$ spatial states $(n-1$ images + particle) after at the $n^{\text {th }}$ spatial reflection through three orthogonal mirrors. There are also 8 possible permutations of these states since one could start with any image and get the same recurrent relations. These $n$ states then transition through the time mirror into past and future conjugate states. One must remember that at any moment, the future thermal energy of such states will according to the second law of thermodynamics be less than the present thermal energy which will be less than the past thermal energy, $E_{f}=k T_{f}<k T<k T_{p}$. This is due to a loss of thermal energy over time. To understand this we look at the transition from one configuration in complex time to another. Thus, the final multiply reflected $n$ spatial states will transition through the complex time mirror say from the present to the past and then from the present to the future will have thermal energy determined by the information transfer function, $\pm i \xi_{t} k T$, where $\xi_{t}$ is complex. If an ensemble of particles is placed in the trap the number of particles transitions from one state to the other say from the present to the future will be given by the 8 image states:

$$
N=8 N_{o} \mathrm{e}^{-\frac{n h v}{i \xi_{t} k T}-\frac{h v}{k T}}
$$

Note that the energy state of the transitions is complex since one is moving from real time to complex time and since the number of particles must be real the variable $\xi_{t}$ must be complex to make this number real. By symmetry, the spatial variables are equal, $\xi_{x}=\xi_{y}=\xi_{z}=\xi_{s}$ and the information density is given by the information wave function and its conjugate after the $k^{\text {th }}$ time transition with a complex time variable $t=i \tau$ :

$$
\psi_{l, m, n}^{k}=8 N_{o}\left(\xi_{s}\right)^{k}\left(\xi_{\tau}\right)^{k} \mathrm{e}^{-\frac{\mathrm{d} E \tau}{\hbar}-\frac{h v}{k T}+\frac{i n h v}{\xi_{t} k T}}
$$

For the frequency interval $\mathrm{d} v$ and time-interval, $\mathrm{d} t=i \mathrm{~d} \tau$, we put $N_{o}=2 \pi(\mathrm{d} v)(i \mathrm{~d} \tau)$. Taking the integral of the absolute value:

$$
\begin{aligned}
& 16 \pi i \iiint \int \frac{v^{3}}{c^{3}} \sum_{n=0}^{\infty}\left(\xi_{\tau}\right)^{2 n} \sum_{n=0}^{\infty}\left(\left(\xi_{s}\right)^{2 n}\right)\left\{\mathrm{e}^{-\frac{\mathrm{d} E \tau}{\hbar}-\frac{h v}{k T}+\frac{i n h v}{\xi_{t} k T}}+\mathrm{e}^{-\frac{\mathrm{d} E \tau}{\hbar}-\frac{h v}{k T}-\frac{i n h v}{\xi_{t} k T}}\right\} \mathrm{d} \lambda^{3} \mathrm{~d} \xi_{\tau}(\mathrm{d} v)(\mathrm{d} \tau)=1, \\
& 16 \pi i \iiint \frac{\frac{v^{3}}{c^{3}} \sum_{n=0}^{\infty} \xi_{s}^{2 n} \cos \xi_{s}^{2 n} \cos \frac{i n h v}{\xi_{t} k T}}{1-\xi_{\tau}^{2}} \mathrm{~d} \lambda^{3} \mathrm{~d} \xi_{\tau}\left(\int_{0}^{\infty} \mathrm{e}^{-\frac{\mathrm{d} E \tau}{\hbar}} \mathrm{d} \tau\right) \mathrm{d} v=1 .
\end{aligned}
$$

Integrating for all time and taking the normalized information density to be unity and if one takes the volume integral,

$$
\int_{0}^{\infty} \mathrm{e}^{-\frac{\mathrm{d} E \tau}{\hbar}} \mathrm{d} \tau=\frac{\hbar}{\mathrm{d} E}
$$

Substituting, $\xi_{\pi}=\frac{i}{x}$ and integrating, we get for the energy density distribution for the frequency range $\mathrm{d} v$;

$$
\mathrm{d} E=\frac{8 h \pi v^{3} \mathrm{e}^{-\frac{h f}{k T}}}{c^{3}} \int_{x=0}^{\infty} \frac{1-\xi_{s}^{2} \cos 1-\xi_{s}^{2} \cos \frac{x h v}{k T}}{\left(1-2 \xi_{s}^{2} \cos 2 \xi_{s}^{2} \cos \frac{x h v}{k T}+\xi_{s}^{2}\right)\left(1+x^{2}\right)} \mathrm{d} x \mathrm{~d} v
$$

and so, 


$$
\mathrm{d} E=\frac{8 \pi v^{3}}{c^{3}} \frac{h v}{\mathrm{e}^{\frac{h f}{k T}}-\xi_{s}^{2}} \mathrm{~d} v
$$

This formula is the Plank's Black Body spectrum for Fermions and bosons. If $\xi_{s}^{2}=1$, the states are bosons, and the particles and the anti-particles states are the same. The reflections of the trap-walls make the particle states and the antiparticle states coexist in same observation space as indistinguishable states. If $\xi_{s}=i$, the particle and its conjugate state are distinct and a double time reflection is needed to bring the particle and anti-particle states into the same observable states. Such is the case for fermions such as electrons and positrons, which transform themselves in the spacetime information trap as antiparticles and particles respectively. However, a double reflection in time is needed to make observation in one trap wall always yields the same state.

\subsection{Symmetry-Maximization Potentials}

I am going to make a statement that has profound implications for our understanding of inflation theory and cosmology.

Statement: The speed of a boson in a logical spacetime continuum is determined by its causal logical determinism.

By this statement I mean the more illogical a quantum system is, the faster it tries to maximize in a space to avoid illogic. Thus a photon has a high speed that will not allow observers to use photons to obtain contradictory and illogical information in a logical space field. Similarly the motions of all bosons are determined by how the spatial motions of observers affect the contradictions of information from that boson in a given logical continuum. Consider a dipole field with perfectly symmetry. Expanding the dipole results in a dynamical system that gives one the Dirac relation. Thus conjugate states are in perfect phase and unobservable as a quantum symmetry. Break the symmetry of the dipole and inflate a pair of mirror spacefields as shown in Figure 22. The initial inflation rate of any point on the trajectory of the inflated spaces close to the vicinity of the dipole can be infinitely fast and no information contradictions can take place. The inflation quickly reduces as the monopoles separate due to the rapid dilution of their contradictions in the space field. I call the speed of information signals in the vacuum the rapidity $\dot{g}$. It is evident that the trajectories of the end points of the extremals joining the object and the image in a mirror will follows the rapidity curves, $x= \pm g(t)$, respectively as shown in Figure 22.

Now such a rapid symmetry break would result in the very definition of the path changing according to some law along symmetric curves $x= \pm g(t)$ since the metric must keep minimizing the integral for each value of the rapidity $\dot{x}^{\mu}$. The present measurement, $\dot{x}^{\mu}$, is the speed of inflation of information in vacuum.

$$
\delta \int_{a(x, j)}^{b(x, j)} \mathrm{d} s=0
$$

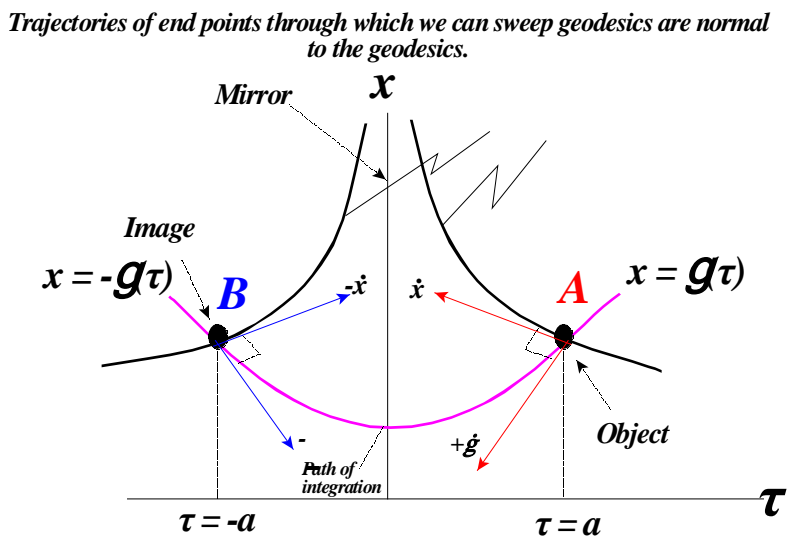

Figure 22. The symmetric potential of matter and antimatter states as a dipole maximizes gives the Dirac relations. 
Imagine the situation of mirror symmetry for the conditions of symmetry of spacetime reflections. One imagines that the trajectories of the information giving states (photons) must necessarily end on the object and image states symmetrically. Thus one has a condition where the end points of the spacetime continuum must follow certain curves. It follows using the theory of variation with variable end points that during the symmetry breaking phase the variation of any conserved variable $E$ between any points $A$ and $B$ whose trajectories lie along curves, $x= \pm g(t)$, respectively satisfy the conditions:

$$
\left.\begin{array}{l}
\text { (i) } \frac{\partial E}{\partial x}-\frac{\mathrm{d}}{\mathrm{d} t}\left(\frac{\partial E}{\partial \dot{x}}\right)=0 \\
\text { (ii) for } t=-a, E+(-\dot{g}-\dot{x})\left(\frac{\partial E}{\partial \dot{x}}\right)=0 \\
\text { (iii) for } t=+a, E+(\dot{g}+\dot{x})\left(\frac{\partial E}{\partial \dot{x}}\right)=0
\end{array}\right\}
$$

If the conditions are not mirror symmetric between $A$ and $B$ then the conditions can be made symmetric again provided one transforms the variables with orthogonal rotation operators $\alpha$ and $\beta$ that will make them symmetric again. These operators are the symmetry restoring rotation matrix operators between the two mirror states and since they are orthogonal and Hermitian they must anti-commute:

$$
\left.\begin{array}{l}
\alpha^{k} \alpha^{l}+\alpha^{l} \alpha^{k}=0, \quad k \neq l \\
\alpha^{k} \beta+\beta \alpha^{k}=0, \\
\left(\alpha^{k}\right)^{2}=(\beta)^{2}=1
\end{array}\right\}
$$

Equations (62) can be written with the matrix operators:

$$
\left.\begin{array}{l}
\text { (i) } \frac{\partial E}{\partial x}-\frac{\mathrm{d}}{\mathrm{d} t}\left(\frac{\partial E}{\partial \dot{x}}\right)=0 \\
\text { (ii) for } t=-a, E-(\beta \dot{g}+\alpha \dot{x})\left(\frac{\partial E}{\partial \dot{x}}\right)=0 \\
\text { (iii) for } t=+a, E+(\beta \dot{g}+\alpha \dot{x})\left(\frac{\partial E}{\partial \dot{x}}\right)=0
\end{array}\right\}
$$

Now considers the integral:

$$
\int_{A}^{B}(H \mathrm{~d} \tau+p \mathrm{~d} x)
$$

where, $H$ and $p$ are functions of $x$ and $\tau$. If the partial first derivatives are continuous functions in the simply connected region of the $(x, \tau)$ plane and

$$
\frac{\partial H}{\partial x}=\frac{\partial p}{\partial \tau}
$$

then, $H \mathrm{~d} \tau=p \mathrm{~d} x=h$, where $h$ is some constant. The path of integration is along the gradient of the extremal (photons) curve and has the compensated gradient relation $\dot{g} \mathrm{~d} t=\mathrm{d} x$. It follows from the theory of variation that the integral (65) is independent of the path of integration and so must be constant. To prove this one substitutes as follows:

$$
H=E-\alpha \dot{x} \frac{\partial E}{\partial \dot{x}}, \quad p=\beta \frac{\partial E}{\partial \dot{x}}
$$

Then

$$
\frac{\partial H}{\partial x}=\left(\frac{\partial E}{\partial x}+\alpha \frac{\partial E}{\partial \dot{x}} \frac{\partial \dot{x}}{\partial x}\right)-\left(\alpha \frac{\partial \dot{x}}{\partial x} \frac{\partial E}{\partial \dot{x}}+\alpha \dot{x} \frac{\partial^{2} E}{\partial \dot{x} \partial x}+\alpha \dot{x} \frac{\partial^{2} E \partial \dot{x}}{\partial \dot{x}^{2} \partial x}\right)
$$


and from $\frac{\partial H}{\partial x}=\frac{\partial p}{\partial \tau}$,

$$
\begin{aligned}
& \frac{\partial H}{\partial x}=\left(\frac{\partial E}{\partial x}-\alpha \dot{x} \frac{\partial^{2} E}{\partial \dot{x} \partial x}-\alpha \dot{x} \frac{\partial^{2} E \partial \dot{x}}{\partial \dot{x}^{2} \partial x}\right)=\left(\beta \frac{\partial^{2} E}{\partial \dot{x} \partial \tau}+\beta \frac{\partial^{2} E \partial \dot{x}}{\partial \dot{x}^{2} \partial \tau}\right) \\
& \text { Since, } \frac{\partial \dot{x}}{\partial \tau}=\alpha \frac{\partial \dot{x}}{\partial \tau}+\beta \frac{\partial \dot{x}}{\partial x} \dot{x}, \frac{\partial E}{\partial x}=\beta \frac{\partial^{2} E}{\partial \dot{x} \partial \tau}+\beta \frac{\partial^{2} E \partial \dot{x}}{\partial \dot{x}^{2} \partial \tau}, \\
& \frac{\partial E}{\partial x}=\left(\beta^{2} \frac{\partial}{\partial \tau}+\alpha^{2} \frac{\partial}{\partial x} \dot{x}\right)\left(\frac{\partial E}{\partial \dot{x}}\right) \\
& \frac{\partial E}{\partial x}=\frac{\mathrm{d}}{\mathrm{d} \tau}\left(\frac{\partial E}{\partial \dot{x}}\right)
\end{aligned}
$$

This leaves the relation (68) independent of the path of integration. It is then clear that in the Spacetime field along the path of integration the integral is invariant and thus the geodesics thus formed are extremals fields. If the velocity variable for the geodesic is the slope of an extremal it follows that:

$$
\left.\begin{array}{l}
\mathrm{e}^{+}=\int_{A}^{B}\left(E+(\beta \dot{g}+\alpha \dot{x})\left(\frac{\partial E}{\partial \dot{x}}\right)\right) \mathrm{d} \tau \\
\mathrm{e}^{-}=\int_{A}^{B}\left(E-(\beta \dot{g}+\alpha \dot{x})\left(\frac{\partial E}{\partial \dot{x}}\right)\right) \mathrm{d} \tau
\end{array}\right\}
$$

is a conserved quantity showing that the charge is conserved. Equation (69) is just the expression for the total relativistic energy of a particle:

$$
\left.\begin{array}{l}
E-\beta m_{o} c-\alpha p c=0 \\
E+\beta m_{o} c+\alpha p c=0
\end{array}\right\}
$$

and Equation (69) is just the Hamiltonian:

$$
H=E-\dot{x} \frac{\partial E}{\partial \dot{x}}
$$

The important fact that arises is that the geodesics requirements for end points of a geodesic that can be moved along extremal curves follow the relativistic Dirac motions. They express the corrections needed to symmetrize a pair of vacuum states such as a positron and an electron due to the spacetime fields. Note that regardless of the velocity fields of the locus the particles will behave as symmetry. The Dirac Spin Matrices now have a clear interpretation as the correction factors needed to symmetrize a particle and its image anti-particle in a dual Spacetime symmetry of matter and antimatter. Note that if the symmetry of the Spacetime was not broken there will exist no metric relations and the velocity fields of the trajectories of the end points will be infinite.

\section{Conclusion}

By this theoretical construction - quantum theory, special theory of relativity, general theory of relativity, the standard model, string theory, mathematics, and the mind can be modeled into one epistemology that is logically consistent with our understanding of classical reality. The concept of information states as logical charges can be extended to massive particles such as electrons and positrons. The Principles of Causal Conspiracy result in epistemologies with far reaching consequences. Quarks are fractional logic systems that cannot be observed in a logical continuum. Nature abhors breaks in symmetry and privileges accorded to information obtained where there was none before observation, must be annihilated back to symmetry according to the Principles of Causal Conspiracy! One can also see that a new basis for considering the Big Bang as a quantum-collapsed state of Matter is possible.

\section{References}

[1] Needham, T. (1997) Visual Complex Analysis. Clarendon Press, Oxford. 
[2] Møller, C. (1952) The Theory of Relativity. 2nd Edition, Oxford University Press, Delhi, 220.

[3] Anthony, M.M. (2013) The Principles of Causal Conspiracy Book 1. Tate Publishing, Mustang.

[4] Baaz, M., Papadimitriou, C., Scott, D., Putnam, H. and Harper, C., Eds. (2011) Kurt Gödel and the Foundations of Mathematics: Horizons of Truth. Cambridge University Press, Cambridge. http://dx.doi.org/10.1017/CBO9780511974236

[5] Genet, C., Intravaia, F., Lambrecht, A. and Reynaud, S. (2004) Electromagnetic Vacuum Fluctuations, Casimir and Van der Waals Forces. Annales de la Fondation Louis de Broglie, 29, 311-328.

[6] Casimir, H.B.G. and Polder, D. (1948) The Influence of Retardation on the London-Van der Waals Forces. Physical Review, 73, 360-372. http://dx.doi.org/10.1103/PhysRev.73.360

[7] Anthony, M.M. (2013) The Principles of Causal Conspiracy Book 2-A Unification of Mind, Matter, and Science. Tate Publishing, Mustang.

[8] Jackson, J.D. and Okun, L.B. (2001) Historical Roots of Gauge Invariance. Reviews of Modern Physics, 73, 663-680. http://dx.doi.org/10.1103/RevModPhys.73.663

[9] Knopp, M. (1970) Modular Functions in Analytic Number Theory. Temple University, Chelsea Publishing Company, Vermont.

[10] Rieger, L. (1951) On Free $\aleph \xi$-Complete Boolean Algebras (with an Application to Logic). Fundamenta Mathematicae, 38, 35-52. 
Scientific Research Publishing (SCIRP) is one of the largest Open Access journal publishers. It is currently publishing more than 200 open access, online, peer-reviewed journals covering a wide range of academic disciplines. SCIRP serves the worldwide academic communities and contributes to the progress and application of science with its publication.

Other selected journals from SCIRP are listed as below. Submit your manuscript to us via either submit@scirp.org or Online Submission Portal.
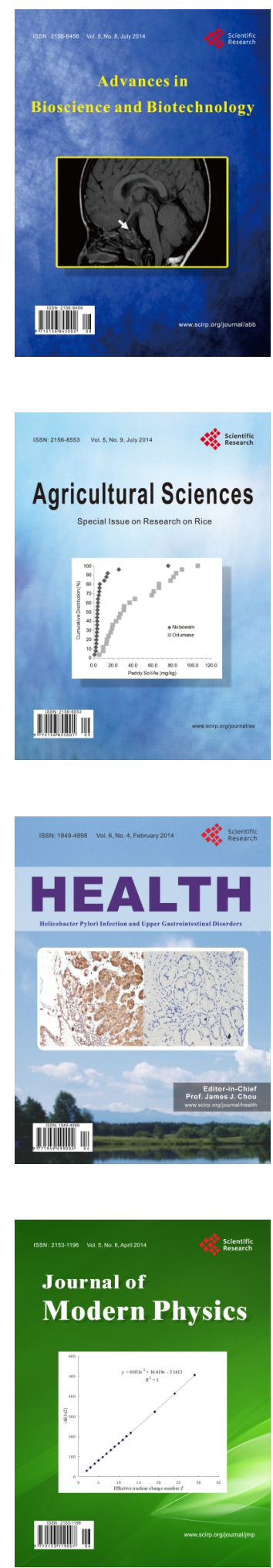
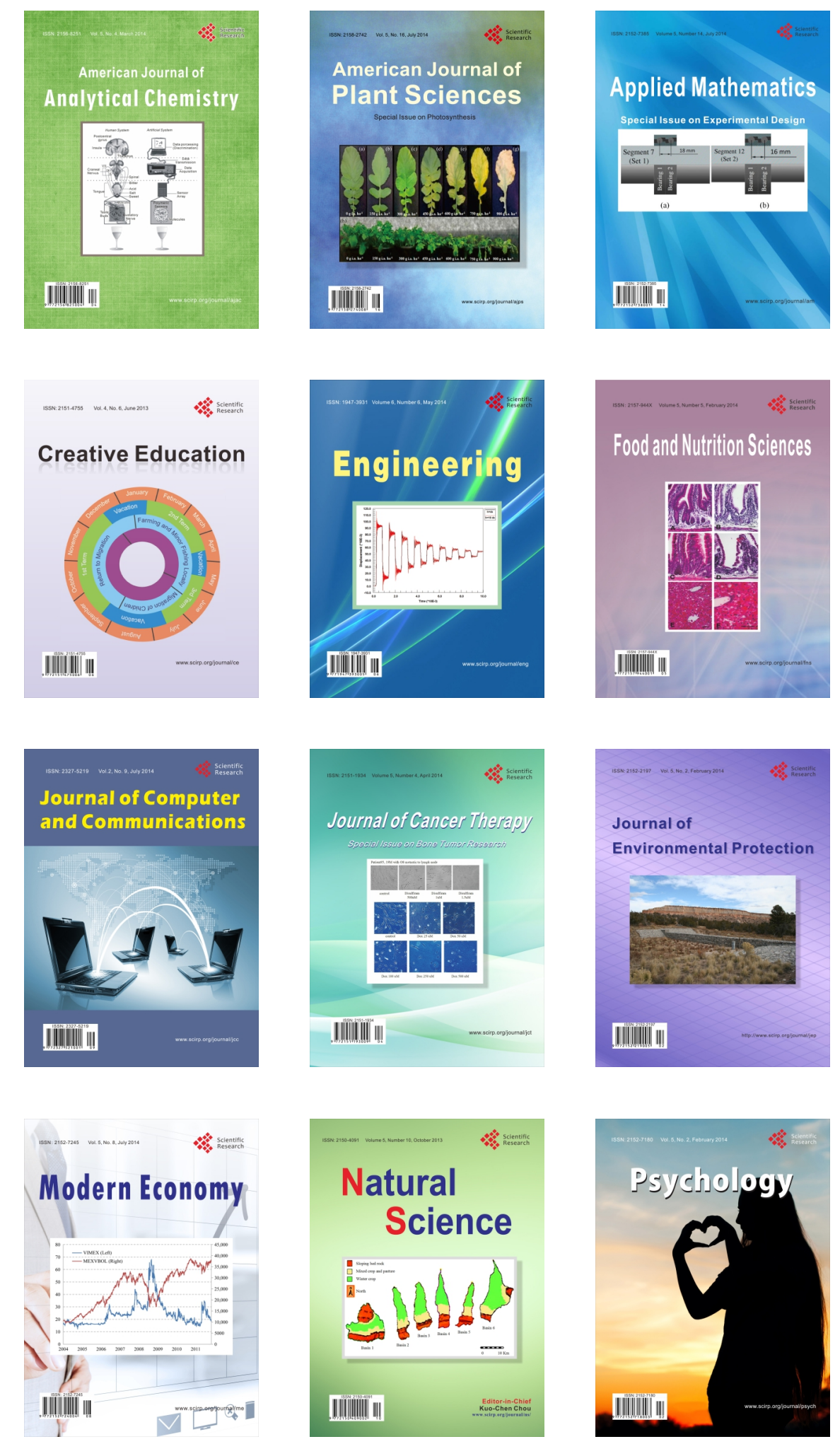